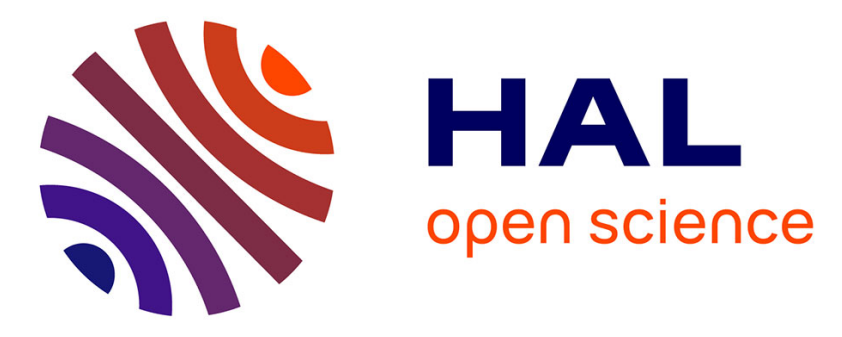

\title{
Glide-Symmetric All-Metal Holey Metasurfaces for Low-Dispersive Artificial Materials: Modeling and Properties
}

Guido Valerio, Fatemeh Ghasemifard, Zvonimir Sipus, Oscar Quevedo-Teruel

\section{- To cite this version:}

Guido Valerio, Fatemeh Ghasemifard, Zvonimir Sipus, Oscar Quevedo-Teruel. Glide-Symmetric All-Metal Holey Metasurfaces for Low-Dispersive Artificial Materials: Modeling and Properties. IEEE Transactions on Microwave Theory and Techniques, 2018, 66, pp.1 - 14. 10.1109/TMTT.2018.2829885 . hal-01805076

\section{HAL Id: hal-01805076 https://hal.sorbonne-universite.fr/hal-01805076}

Submitted on 12 Mar 2020

HAL is a multi-disciplinary open access archive for the deposit and dissemination of scientific research documents, whether they are published or not. The documents may come from teaching and research institutions in France or abroad, or from public or private research centers.
L'archive ouverte pluridisciplinaire HAL, est destinée au dépôt et à la diffusion de documents scientifiques de niveau recherche, publiés ou non, émanant des établissements d'enseignement et de recherche français ou étrangers, des laboratoires publics ou privés. 


\title{
Glide-Symmetric All-Metal Holey Metasurfaces for Low-Dispersive Artificial Materials: Modelling and Properties
}

\author{
Guido Valerio, Member, IEEE, Fatemeh Ghasemifard, Student Member, IEEE, \\ Zvonimir Sipus, Senior Member, IEEE, and Oscar Quevedo-Teruel, Senior Member, IEEE
}

\begin{abstract}
We study the wave propagation between two glidesymmetric metallic plates drilled with periodic rectangular holes. A mode-matching method is proposed in order to derive efficiently the dispersive properties of these periodic structures. The method takes advantage of the higher symmetry of the structure reducing the computational cost by enforcing boundary conditions on the field on only one of the two surfaces. Physical insight on specific symmetry properties of Floquet harmonics in glide-symmetric structures is also gained. The code is validated with commercial software assessing its accuracy when varying the most influential/critical parameters. We confirm the potential of glide-symmetric structures to tune the effective refractive index. Specifically, we demonstrate that glide-symmetric structures with rectangular shapes can be employed to synthesize anisotropic refractive indexes with a large band of operation, which makes such metasurface structures applicable for realization of UWB planar lenses.
\end{abstract}

Index Terms-Metasurfaces, glide symmetry, higher symmetries, periodic structures, mode matching, numerical methods, dispersive analyses.

\section{INTRODUCTION}

A RTIFICIAL materials made by periodic lattices are an established solution to control wave-propagation features of Bloch modes leading to rich properties for guided and radiating waves [1]-[5]. After the intense work of the last decades, recent research in the area of metamaterials and metasurfaces aims at the synthesis of desired dispersion diagrams. For instance, protected propagation of confined edge waves between artificial materials can be obtained with non-trivial topological dispersion surfaces [6], [7]. Extreme frequency dispersion can be obtained by suitably coupling different periodic lines [8], [9], with applications to accelerators and compact resonators. These results are achieved by adding finer

This work was supported in part by the Spanish Government under Project TEC2013-44019-R, by the Swedish STINT Postdoctoral Transition Grants programme under Grant PT2014-5813 funded by the Swedish Foundation for International Cooperation in Research and Higher Education, by the French ANR grant HOLeYMETA ANR-16-CE24-0030, and by the French "Sorbonne Universités" grant MetaSym.

G. Valerio is with Sorbonne Université, UR2, L2E, F-75005 Paris, France. e-mail: (guido.valerio@sorbonne-universite.fr).

Z. Sipus is with with the Faculty of Electrical Engineering and Computing, University of Zagreb, Unska 3/XII, HR-10000 Zagreb, Croatia. e-mail: (zvonimir.sipus@fer.hr).

F. Ghasemifard and O. Quevedo-Teruel are with with the Department of Electromagnetic Engineering, School of Electrical Engineering, KTH Royal Institute of Technology, SE-100 44, Sweden. e-mail: (fatemehg@kth.se, oscarqt@kth.se).

Manuscript received Mai 1, 2017; revised Mai 2, 2017. details to the inner structure of each unit cell of periodicity [10].

Specifically, symmetries can provide a number of interesting dispersive effects. They can be defined with respect either to space or time operators. The latter requires a lattice alternating lossy and gain scatterers [11] and are not practical for current low-cost and low-loss devices in mass production.

Instead, the presence of higher symmetries involving only spatial operations can simplify the practical realizations of low-cost devices, and still provide very interesting dispersive properties not commonly find in periodic structures. Spatial higher symmetries in periodic structures can be of different kinds: the most used in electromagnetics are the twist and the glide types [12], [13]. While the former involves a symmetry under a translation followed by a rotation, the latter involves a symmetry under a translation followed by a mirror, thus being compatible with fully planar geometries of use in mm-wave components and integrated-circuit technology [14], [15].

More specifically, the glide operator is a geometrical transformation composed by a translation along one or more directions and a mirroring with respect to the so-called glide plane. To fix the ideas, in Fig. 1(a), two 2-D glide-symmetric metallic surfaces with squared holes are shown. They are invariant under the glide operation $G_{d}$ :

$$
\left\{\begin{array}{clc}
(x, y) & \rightarrow & \left(x+\frac{d}{2}, y+\frac{d}{2}\right) \\
z & \rightarrow & -z
\end{array}\right.
$$

if the $z=0$ plane is in the middle of the two surfaces. Note, $G_{d}^{2}=T_{d}$, where $T_{d}$ is a translation of $d$ along both $x$ and $y$. For this reason, a glide-symmetric structure is also periodic. The unit cell of Fig. 1(a) is given in Fig. 1(b). When rectangular-shape holes are assumed, the cell is extended to the one represented in in Fig. 1(c).

Higher symmetries were at first studied decades ago in connection to the theory of periodic waveguides [12], [16][19], and a generalized Floquet theorem was stated in [12]. The interest for glide symmetric structures recently renewed after several years in connection with the recent development of metamaterials and metasurfaces related to backward propagation [20], leaky radiation [21] and artificial materials [22], [23]. The first 2-D glide-symmetric metasurface for antennas was proposed in [14] (Fig. 1(a)) and used to implement a UWB flat Luneburg lens. The glide symmetry of the unit cell enables the propagation of a wave having a linearly varying 
phase constant in an ultrawide band (UWB) of frequencies. This means that a remarkable absence of frequency dispersion can be observed, and the equivalent refractive index is constant over an UWB range of frequencies. Furthermore, even in the absence of a dielectric between the holey surfaces, the equivalent refractive index is dependent on several geometric parameters [24]. By tuning their values, this refractive index can also be easily varied along the surface in an adiabatic way [25] -[31], thus obtaining the index variation required to realize a Luneburg lens as in [14]. These properties make glidesymmetric metasurfaces ideal candidates for UWB low-loss all-metal graded-index millimeter-wave lenses: a long-desired feature for the design of devices as increasingly required by next generation satellite and terrestrial communication standards [32],[33]. Other glide-symmetric topologies confirm these UWB properties in 1-D configurations [34], [35]. While the absence of frequency dispersion can be observed at low frequencies, the presence of a doubly periodic load on the two surfaces has the effect to enhance the width of the stop band present at higher frequencies. This phenomenon opens an opportunity for the design of electromagnetic band gap (EBG) materials for mm-band components and integrated technology, which exploit the presence of a very wide stopband for efficient field confinement. In [15],[36],[37] these devices have been designed, fabricated and measured, thus proving the possibility to exploit this stop-band behavior for practical applications.

Due to the particular kind of symmetry defined in (1), the fabrication of glide-symmetric prototypes, as explained in [15], is done in two separated pieces, which are the two symmetric holey plates. These pieces require a specific alignment between them, since their reciprocal shift must be of half a period along all the periodicity directions. Since the plates require no dielectric in between them, they are joined together with lateral dielectric screws, which can tune the required gap. The alignment is further controlled by means of alignment vias placed at the side of the structure [24]. The feasibility of such a manufacturing process has recently been demonstrated in [13],[15],[34],[35] where measurements have confirmed the observation of the dispersive phenomena predicted by the Floquet theory used here. For this reason, fabrication and measurement issues are not in the focus in this paper, which is rather aimed at studying the properties of Floquet harmonics in glide-symmetric surfaces and a modeling technique exploiting the higher symmetry.

In fact, the novelty of these structures stimulates the research of simple modeling methods to describe their properties. Even if one single corrugated surface, as depicted in Fig. 2(a), can be described with a homogenized impedance [38], [39], this model does not hold in the presence of two strongly interacting surfaces as in a glide-symmetric configuration. The problem was recently addressed in [40] by means of an equivalent circuit, where an interpretation of the effect of the glide-symmetry is given in term of an effective division by half of the period. Furthermore, the structures of interest for graded-index lenses applications, as in [14], are characterized by a very large ratio size of the hole vs. gap between surfaces. Under these conditions, conventional techniques are very inefficient due to unusual field variations with respect to common holey and corrugated structures. Both commercial and ad-hoc software face convergence problems due to the presence of the very thin air layer between the surfaces.

In this paper, we propose a mode-matching technique [41],[42] in order to derive efficiently the dispersive features of glide-symmetric corrugated and holey surfaces. While modematching methods have been proposed for corrugated structures in the absence of glide symmetry [43] and for doublycorrugated structures [44], [45], the present method for the first time uses the higher symmetry of the structure in order to reduce the computational domain to one half of the unit cell. The results presented in [43]-[45] show that a mode matching method is more efficient than commercial software based on finite-method algorithms [46] when the ratio size of the corrugation/hole vs. gap between surfaces is large. Furthermore, while other integral-equation-based approaches could also be attempted [47]-[51], the present mode matching provides physical insight on specific symmetry properties of Floquet harmonics propagating in glide-symmetric structures, and gives information on the behavior of the field excited inside the holes. The code is validated by comparing the results with commercial software. A large number of parametric analyses validate the code and prove the possibility to simply tuning the effective refractive index by selecting appropriate values of relevant geometric parameters. A specific study is performed for the first time on holes with rectangular shape, which are proved to synthesize different equivalent refractive index for wave propagation along the two principal axes. The expected degeneracy of the eigenvalues at the edge of each Brillouin zone is recovered, and a lack of frequency dispersion on a ultra-wide range of frequencies is confirmed by all the numerical results. This study will enable the design of all-metal glide-symmetric metasurfaces for UWB anisotropic wave propagation, to be easily employed for the fabrication of low-cost and low-loss graded-index planar lenses.

In Section II, we present the formulation of the mode matching, first in the corrugated geometry in Fig. 2 (1D glidesymmetric structure), and after for the holey geometry in Fig. 1 (2D glide-symmetric structure). In Section III, numerical results validate the analysis, recovering the fundamental spectral properties of glide-symmetric structures, and show the effect of parameter variations on the effective refractive index achieved, both in the case of isotropic and anisotropic surfaces.

\section{Formulation OF THE PROBLEM}

In this section we present a mode-matching formulation exploiting the peculiar properties of glide symmetry in metallic metasurfaces as depicted in Fig. 1(a) and 2(a). The aim of the study is performing a dispersive analysis of the structure, i.e., computing the wavenumber of modes propagating between the glide-symmetric surfaces along an arbitrary direction. The computation of the wavenumber in a certain range of frequencies will give information on the equivalent refractive index seen by the wave and its frequency dispersive properties (frequency variations). Furthermore, a glide-symmetric mode matching will give a physical insight about the presence of 


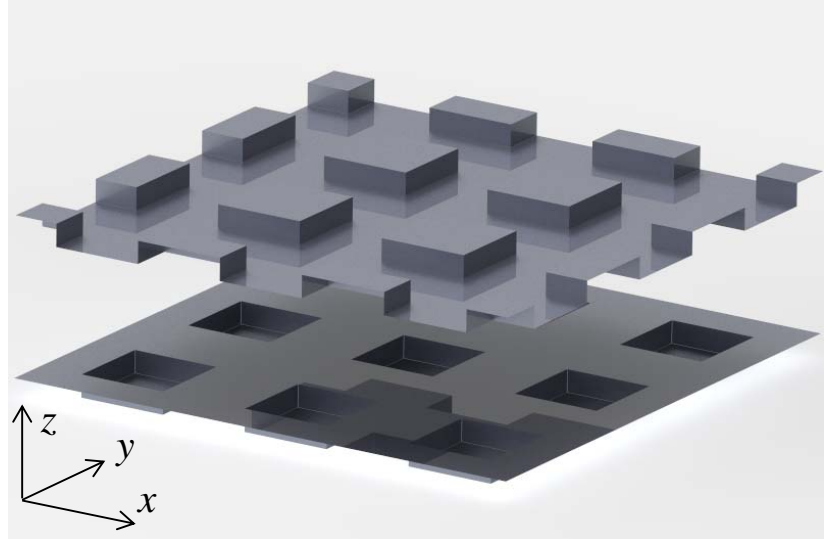

(a)

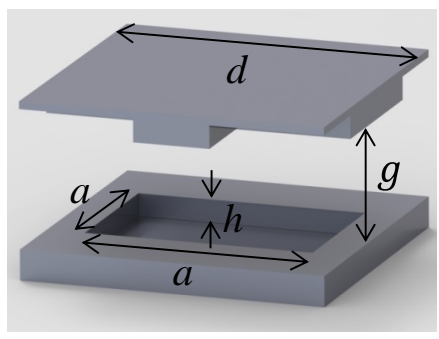

(b)

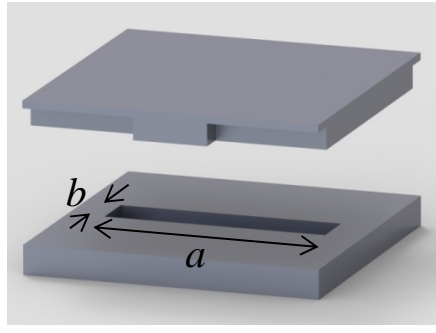

(c)
Fig. 1. 2-D periodic holey metallic plates with glide symmetry: (a) glidesymmetric surfaces, (b) unit cell with squared hole, (c) unit cell with rectangular hole (having lateral sizes $a$ and $b$ ).

additional symmetry properties of different Floquet harmonics inside the waveguide, as a consequence of the additional symmetry of the periodic unit cell. Finally, the proposed analysis requires the use of a small number of degrees of freedom on one hole of one surface only. In fact, the glide symmetry is taken into account by a proper symmetry of each harmonics and the boundary conditions do not need to be enforced on both surfaces. This approach is much faster than meshing the whole periodic unit cell as in a commercial software, especially in the structures of interest where the aspect ratio $a / g$ is very large (around 30) and very small cells are required to describe the field variation between the surfaces.

The two glide-symmetric metallic plates are placed at $z=-g / 2$ and at $z=g / 2$. Since boundary conditions will be enforced on horizontal components of fields (where the vertical direction is the $z$ in Fig. 1 and 2), vertical fields will not be explicitly computed here. The transverse components of fields (on the $x y$ plane) will be indicated with the subscript ' $\mathrm{t}$ '.

\section{A. The generalized Floquet's theorem}

The standard Floquet theorem for periodic structures states that a Bloch mode is an eigenmode of the translation operation $T_{d}$ of length $d$ along $x$ : the field is periodic across one unit cell apart from an exponential factor describing propagation and/or attenuation:

$$
\mathbf{E}(x+d, y, z)=T_{d}[\mathbf{E}(x, y, z)]=e^{-j k_{x} d} \mathbf{E}(x, y, z),
$$

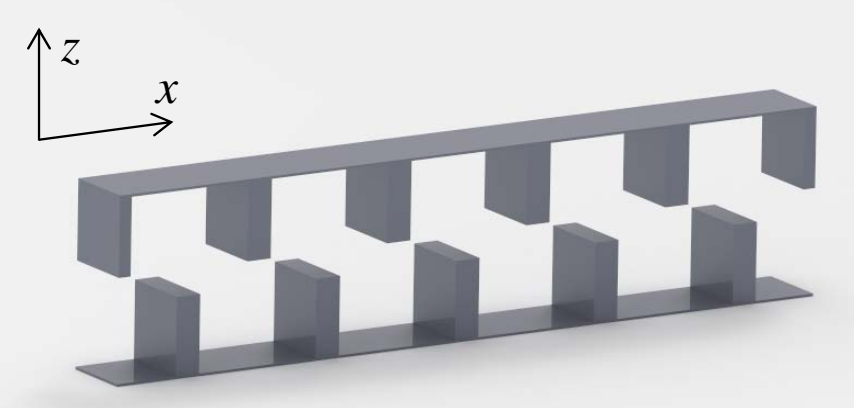

(a)

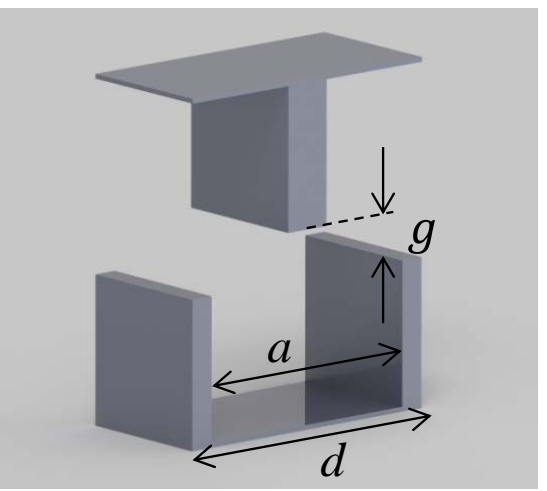

(b)

Fig. 2. 1-D periodic holey metallic corrugations: (a) glide-symmetric surfaces, (b) unit cell.

where $k_{x}=k_{x, 0}+2 \pi p / d$ and $p$ is an integer.

The generalized theorem states that a Bloch mode of a glidesymmetric structure is not only an eigenmode of the translation operator $T_{d}$, but also of the glide operator $G_{d}$. In other words, after a translation of half a period and a mirroring operation, the field repeats itself apart from an exponential factor:

$$
\mathbf{E}\left(x+\frac{d}{2}, y,-z\right)=G_{d}[\mathbf{E}(x, y, z)]= \pm e^{-j k_{x, 0}^{ \pm} \frac{d}{2}} \mathbf{E}(x, y, z)
$$

so that a the application of two glide operators is consistent with the periodicity condition (2).

It should be remarked that the double sign in front of the exponential does not introduce any ambiguity, neither it defines two different sets of modes. In fact, it is easy to verify that, if $k_{x, 0}^{+}$and $\mathbf{E}$ are solutions of (3) with the plus sign, then $k_{x, 0}^{-}=k_{x, 0}^{+}+2 \pi / d$ and the same field $\mathbf{E}$ are solutions of (3) with the minus sign. Hence, the solutions of the two problems coincide, apart a re-labeling of the harmonics: the harmonic 0 becomes the harmonic -1 when switching the sign from plus to minus. Since this relabeling does not have any physical meaning, in the following only the plus sign will be considered and $k_{x, 0}^{+}$will be simply named $k_{x, 0}$.

The spatial property (3) is responsible of the other important spectral property of glide-symmetric structures. The $\mathrm{X}$ point in the Brillouin zone $\left(k_{x, 0}=\pi / d, x\right.$ being the direction of glide symmetry) is a degenerate point, where two real solutions merge at the same frequency without the presence of a stop 
band. In other words, glide symmetry suppresses stop-band at even multiple of $\pi / d$ ("closed stop band").

\section{B. Glide-symmetric corrugated surfaces}

In this section, glide-symmetric corrugated surface illustrated in Fig. 2 is analysed using mode matching technique. The structure is periodic along $x$, invariant along $y$ and bounded along $z$. The gap between two layers is $g$ and the plane $z=0$ is located in the middle of the gap. Since structure is invariant in the $y$-direction, the $y$-dependence of the fields is written as $\exp \left(-j k_{y} y\right)$ and omitted. $\mathrm{TE}_{y}$-modes with $E_{y}=0$ are the first dominant modes that can propagate in this structure. Considering these modes, the tangential fields that need to be matched are $E_{x}, H_{x}$, and $H_{y}$. However, $H_{x} \propto \partial H_{y} / \partial x$ which means continuity in $z$ of $H_{y}$ implies continuity in $z$ of $H_{x}$. Thus, only $E_{x}$ and $H_{y}$ need to be matched.

In the gap region between the upper and the lower layer, $E_{x}^{\text {Gap }}$ and $H_{y}^{\text {Gap }}$ can be expressed as a series of Floquetharmonics by virtue of periodicity:

$$
\begin{aligned}
& E_{x}^{\mathrm{Gap}}=\frac{1}{d} \sum_{p} e^{-j k_{x, p} x}\left[A_{p}^{x} \sin \left(k_{z, p} z\right)+B_{p}^{x} \cos \left(k_{z, p} z\right)\right] \\
& H_{y}^{\mathrm{Gap}}=\frac{1}{d} \sum_{p} e^{-j k_{x, p} x}\left[D_{p}^{y} \sin \left(k_{z, p} z\right)+F_{p}^{y} \cos \left(k_{z, p} z\right)\right]
\end{aligned}
$$

where $k_{x, p}=k_{x, 0}+2 \pi p / d$ and $k_{z, p}=\sqrt{k_{0}^{2}-k_{y}^{2}-k_{x, p}^{2}}$.

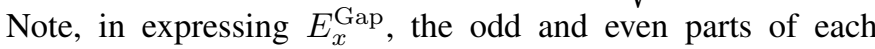
harmonic along $z$ with respect to the middle plane of the gap region $(z=0)$ have been explicitly written into two different terms. Their amplitudes are the $A$ and $B$ coefficients, respectively. The sums in (4) and (5) are extended to all integer values of $p$. Its truncation for numerical calculation purposes will be described at the end of the next subsection.

Using source-free Maxwell equations $\nabla \cdot \mathbf{E}^{\mathrm{Gap}}=0$ and $\nabla \times \mathbf{E}^{\mathrm{Gap}}=-j \omega \mu_{0} \mathbf{H}^{\mathrm{Gap}}$, we have

$$
\begin{aligned}
& \eta_{0} D_{p}^{y}=\frac{1}{j k_{0}} B_{p}^{x} \frac{k_{0}^{2}-k_{y}^{2}}{k_{z, p}} \\
& \eta_{0} F_{p}^{y}=\frac{-1}{j k_{0}} A_{p}^{x} \frac{k_{0}^{2}-k_{y}^{2}}{k_{z, p}} .
\end{aligned}
$$

Note that the magnetic-field quantities have been normalized to the free-space impedance $\eta_{0}$ to simplify some equations.

As the next step, to enforce the continuity of $E_{x}$ and $H_{y}$ among the two regions, their expression in each corrugation must be written. A suitable way to do so is a modal expansion of parallel plate waveguide (PPW) modes. Each corrugation in the lower surface can be regarded as a short-circuited parallel plate waveguide with length $h$ along the $z$ axis. Therefore, on the plane $z=-g / 2$, the $x$-component of the electric field named $E_{x}^{\mathrm{PPW}}$ and the $y$-component of the magnetic field named $H_{y}^{\mathrm{PPW}}$ can be expressed, respectively, as

$$
\begin{aligned}
E_{x}^{\mathrm{PPW}}\left(x, z=-\frac{g}{2}\right) & =\sum_{m} r_{m}^{-} C_{m} \Phi_{m}(x) \\
\eta_{0} H_{y}^{\mathrm{PPW}}\left(x, z=-\frac{g}{2}\right) & =\frac{k_{0}^{2} \varepsilon_{\mathrm{r}}-k_{y}^{2}}{k_{0}} \sum_{m} \frac{r_{m}^{+}}{q_{m}} C_{m} \Phi_{m}(x)
\end{aligned}
$$

over $0<x<a$ and $E_{x}^{\mathrm{PPW}}(x, z=-g / 2)=0$ over $a<x<$ $d$. In (7) and (8), the $C_{m}$ coefficients are the undetermined amplitudes of each mode,

$$
\Phi_{m}(x)=\left\{\begin{array}{cc}
\sqrt{\frac{2}{a}} \cos \left(m \pi \frac{x}{a}\right) & m \neq 0 \\
\sqrt{\frac{1}{a}} & m=0
\end{array}\right.
$$

are the normalized modal functions, and

$$
r_{m}^{ \pm}=1 \pm e^{-j 2 q_{z, m} h}
$$

are the magnetic-field and electric-field reflection coefficients due to the short circuit at the end of the corrugation. Note, $a$ is the width of the corrugation, $\epsilon_{\mathrm{r}}$ is the dielectric constant of the material inside the hole, and $q_{z, m}=\sqrt{\epsilon_{\mathrm{r}} k_{0}^{2}-k_{y}^{2}-m^{2} \pi^{2} / a^{2}}$ is the wavenumber of the $m$-th mode.

\section{Glide-symmetric boundary conditions for the $1 D$ structure}

In this subsection, we enforce boundary conditions at the connection between the gap region and each corrugation, in order to obtain a dispersion equation to be numerically solved for the wavenumbers of Bloch modes supported by the structure.

Thanks to the periodicity of the structure, we can enforce boundary conditions only inside one unit cell. This requires to formulate a boundary condition on the corrugation in the both lower surface $(z=-g / 2,0<x<a)$ and upper surface $(z=g / 2, d / 2<x<a+d / 2)$. Restricting the analysis to a unit cell is justified since the Bloch modes are eigenmodes of the translation operator $T_{d}$, so that the fields in two adjacent unit cells are proportional through the propagation factor $\exp \left(-j\left(k_{x, 0} d+k_{y, 0} d\right)\right)$.

However, the generalized Floquet theorem discussed in [12] allows us to further simplify the analysis and reduce the number of independent boundary conditions to be formulated. The theorem states that a Bloch mode of the glide-symmetric structure is not only an eigenmode of the translation operator $T_{d}$, but also of the glide operator $G_{d}$. In other words, after a translation of half a period and a mirroring around the glide plane $(z=0)$ the field is the same, apart a factor $\pm \exp \left(-j k_{x, 0} d / 2\right)$, where the \pm sign depends on the Floquet harmonic considered (without loss of generality, we continue the formulation with the plus sign). This factor allows to recover the correct phasing when obtaining a translation of a period as the composition of two subsequent glide transformations.

Now, enforcing the continuity of the electric field on the lower surface $(z=-g / 2,0<x<a)$, we have:

$$
E_{x}^{\mathrm{Gap}}\left(x, z=-\frac{g}{2}\right)=E_{x}^{\mathrm{PPW}}\left(x, z=-\frac{g}{2}\right) .
$$


On the upper surface $(z=g / 2, d / 2<x<a+d / 2)$, by means of glide-symmetry we can write,

$$
E_{x}^{\mathrm{Gap}}\left(x, z=\frac{g}{2}\right)=e^{-j k_{x, 0} \frac{d}{2}} E_{x}^{\mathrm{PPW}}\left(x-\frac{d}{2}, z=-\frac{g}{2}\right) .
$$

When the Floquet series (4) is replaced into (11) and (12) and inverted, we obtain, respectively,

$$
\begin{aligned}
-A_{p}^{x} \sin \left(\theta_{z, p}\right)+B_{p}^{x} \cos \left(\theta_{z, p}\right) & =\tilde{E}_{x}^{\mathrm{PPW}}\left(k_{x, p}\right), \\
A_{p}^{x} \sin \left(\theta_{z, p}\right)+B_{p}^{x} \cos \left(\theta_{z, p}\right) & =(-1)^{p} \tilde{E}_{x}^{\mathrm{PPW}}\left(k_{x, p}\right),
\end{aligned}
$$

where $\theta_{z, p}=k_{z, p} g / 2$,

$$
\tilde{E}_{x}^{\mathrm{PPW}}\left(k_{x, p}\right)=\sum_{m} r_{m}^{-} C_{m} \tilde{\Phi}_{m}\left(k_{x, p}\right)
$$

is the Fourier transforms of $E_{x}^{\mathrm{PPW}}(x, z=-g / 2)$ expressed in (7), i.e.

$$
\tilde{\Phi}_{m}\left(k_{x, p}\right)=\int_{0}^{a} \Phi_{m}(x) e^{j k_{x, p} x} d x
$$

are the Fourier transforms of the modal functions $\Phi_{m}(x)$. Equations (13) and (14) show an interesting symmetry property of Floquet harmonics in glide-symmetric structures.

If $p$ is odd:

$$
\begin{aligned}
A_{p}^{x} & =-\frac{\tilde{E}_{x}^{\mathrm{PPW}}\left(k_{x, p}\right)}{\sin \left(k_{z, p} g / 2\right)} \\
B_{p}^{x} & =0
\end{aligned}
$$

If $p$ is even:

$$
\begin{aligned}
A_{p}^{x} & =0 \\
B_{p}^{x} & =\frac{\tilde{E}_{x}^{\mathrm{PPW}}\left(k_{x, p}\right)}{\cos \left(k_{z, p} g / 2\right)} .
\end{aligned}
$$

We can see that even (odd) harmonics have an even (odd) transverse electric field along the $z$ direction with respect to the plane $z=0$, which is called the glide plane.

Rewriting $H_{y}^{\text {Gap }}$ on the surface $z=-g / 2$ using (5), (6), (17), and (18), we obtain:

$$
\begin{aligned}
& \eta_{0} H_{y}^{\text {Gap }}\left(x, z=-\frac{g}{2}\right) \\
& =\frac{k_{0}^{2}-k_{y}^{2}}{j k_{0} d} \sum_{p} e^{-j k_{x, p} x} \frac{\tilde{f}_{p}}{k_{z, p}} \sum_{m} r_{m}^{-} C_{m} \tilde{\Phi}_{m}\left(k_{x, p}\right),
\end{aligned}
$$

where

$$
\tilde{f}_{p}=\left\{\begin{array}{ccc}
-\tan \left(\frac{k_{z, p} g}{2}\right) & p & \text { even } \\
\cot \left(\frac{k_{z, p} g}{2}\right) & p & \text { odd }
\end{array}\right.
$$

is called here the vertical spectral function. Indeed, this function defines the glide-symmetry of the structure. A different stratified configuration (such as the unbounded air or a metal plate on the top of a single corrugated surface) would only require a different definition of $\tilde{f}_{p}$.

Now, we can see that the only $C_{m}$ coefficients are undetermined. Thus, imposing boundary condition for $H_{y}$ on one surface is enough to achieve the dispersion equation. Indeed, after imposing the continuity of $H_{y}$ on one surface $(z=-g / 2)$, the continuity condition on the other surface $(z=g / 2)$ is automatically verified thanks to the symmetry properties of Floquet harmonics in glide-symmetric structures.
Enforcing the continuity of the magnetic fields (8) and (19) across the corrugation aperture and projecting this equation on each waveguide modal function $\Phi_{m^{\prime}}(x)$ (multiplying both sides of the equation by $\Phi_{m^{\prime}}(x)$ and integrating over the corrugation aperture), the following linear equations are obtained:

$$
\sum_{m} \alpha_{m^{\prime}, m} C_{m}=0
$$

where

$$
\begin{aligned}
\alpha_{m^{\prime}, m} & =\frac{k_{0}^{2}-k_{y}^{2}}{j d} r_{m}^{-} \sum_{p} \tilde{\Phi}_{m}\left(k_{x, p}\right) \tilde{\Phi}_{m^{\prime}}\left(-k_{x, p}\right) \frac{\tilde{f}_{p}}{k_{z, p}} \\
& -\delta_{m^{\prime}, m}\left(k_{0}^{2} \varepsilon_{\mathrm{r}}-k_{y}^{2}\right) \frac{r_{m}^{+}}{q_{z, m}},
\end{aligned}
$$

and $\delta_{m^{\prime}, m}$ is zero unless $m=m^{\prime}$. Rewriting (22) for the case that $k_{y}=0, \varepsilon_{\mathrm{r}}=1$, and only one modal function $\Phi$ is taken into account, we obtain the dispersion equation

$$
\begin{aligned}
\frac{j d}{q_{z, 0}} \frac{r^{+}}{r^{-}} & =\sum_{p \text { odd }} \tilde{\Phi}\left(k_{x, p}\right) \tilde{\Phi}\left(-k_{x, p}\right) \frac{\cot \left(k_{z, p} g / 2\right)}{k_{z, p}} \\
& -\sum_{p \text { even }} \tilde{\Phi}\left(k_{x, p}\right) \tilde{\Phi}\left(-k_{x, p}\right) \frac{\tan \left(k_{z, p} g / 2\right)}{k_{z, p}} .
\end{aligned}
$$

This equation shows the symmetry properties of different harmonics in a glide-symmetric structure compared to the dispersion relation of a corrugated surface in the air [52]. Furthermore, it is easy to realize that if $g \rightarrow+\infty$ and all $k_{z, p}$ are imaginary (i.e., $k_{z, p}=-j\left|k_{z, p}\right|$ for bound modes), (23) reduces to the dispersion equation of a corrugated surface in free space presented in [52].

It is remarkable that the truncation of summations in (4) and (7) for numerical implementation has some implications. If in (4) and (7), the summations are truncated, respectively, at $p=$ $P$ and $m=M$, the most rapid modes that need to be matched on horizontal plates $z= \pm g / 2$ are $\exp (-j(2 P+1) \pi x / d)$ and $\cos (M \pi x / a)$. Thus, the optimal value for the number of modes in each region can be obtained from $M \pi / a=(2 P+$ $1) \pi / d$ or equivalently $M /(2 P+1)=a / d$. In other words, the ratio of PPW modes to Floquet modes should be almost equal to the ratio $a / d$.

\section{Glide-symmetric holey surfaces}

In this section, we introduce a mode matching technique for analysing the glide-symmetric holey surface (2D periodic structure) illustrated in Fig. 1. The formulation is very similar to the one presented for the glide-symmetric corrugated surface (1D periodic structure).

The fields in the gap region $\mathbf{E}_{\mathrm{t}}^{\mathrm{Gap}}$ and $\mathbf{H}_{\mathrm{t}}^{\mathrm{Gap}}$ can be expressed as:

$$
\begin{aligned}
\mathbf{E}_{\mathrm{t}}^{\mathrm{Gap}} & =\frac{1}{d^{2}} \sum_{p q} e^{-j\left(k_{x, p} x+k_{y, q} y\right)} \tilde{\mathbf{e}}_{\mathrm{t}, p q}^{\mathrm{Gap}}(z) \\
\mathbf{H}_{\mathrm{t}}^{\mathrm{Gap}} & =\frac{1}{d^{2}} \sum_{p q} e^{-j\left(k_{x, p} x+k_{y, q} y\right)} \tilde{\mathbf{h}}_{\mathrm{t}, p q}^{\mathrm{Gap}}(z)
\end{aligned}
$$

with $k_{x, p}=k_{x, 0}+2 \pi p / d$ and $k_{y, q}=k_{y, 0}+2 \pi p / d$ by assuming a rectangular lattice. The sum is extended to all integer 
values of $p$ and $q$. Its truncation for numerical calculation purposes will be described in Section III. The amplitude of each Floquet harmonic of the transverse electric field can be written, with no loss of generality, as

$$
\tilde{\mathbf{e}}_{\mathrm{t}, p q}^{\mathrm{Gap}}(z)=\left(\begin{array}{c}
A_{p q}^{x} \\
A_{p q}^{y}
\end{array}\right) \sin \left(k_{z, p q} z\right)+\left(\begin{array}{c}
B_{p q}^{x} \\
B_{p q}^{y}
\end{array}\right) \cos \left(k_{z, p q} z\right)
$$

where $k_{z, p q}=\sqrt{k_{0}^{2}-k_{x, p}^{2}-k_{y, q}^{2}}$ is the vertical wavenumber of the $(p, q)$ th harmonic.

The transverse components of the magnetic-field harmonics $\tilde{\mathbf{h}}_{\mathrm{t}, p q}^{\text {Gap }}$ can be easily derived from (24) and (26) in a similar way as $1 \mathrm{D}$ case:

$$
\tilde{\mathbf{h}}_{\mathrm{t}, p q}^{\mathrm{Gap}}(z)=\left(\begin{array}{c}
D_{p q}^{x} \\
D_{p q}^{y}
\end{array}\right) \sin \left(k_{z, p q} z\right)+\left(\begin{array}{c}
F_{p q}^{x} \\
F_{p q}^{y}
\end{array}\right) \cos \left(k_{z, p q} z\right),
$$

where

$$
\begin{aligned}
& \eta_{0} D_{p q}^{x}=\frac{-1}{j k_{0}}\left(B_{p q}^{x} \frac{k_{x, p} k_{y, q}}{k_{z, p q}}+B_{p q}^{y} \frac{k_{0}^{2}-k_{x, p}^{2}}{k_{z, p q}}\right) \\
& \eta_{0} D_{p q}^{y}=\frac{1}{j k_{0}}\left(B_{p q}^{x} \frac{k_{0}^{2}-k_{y, q}^{2}}{k_{z, p q}}+B_{p q}^{y} \frac{k_{x, p} k_{y, q}}{k_{z, p q}}\right) \\
& \eta_{0} F_{p q}^{x}=\frac{1}{j k_{0}}\left(A_{p q}^{x} \frac{k_{x, p} k_{y, q}}{k_{z, p q}}+A_{p q}^{y} \frac{k_{0}^{2}-k_{x, p}^{2}}{k_{z, p q}}\right) \\
& \eta_{0} F_{p q}^{y}=\frac{-1}{j k_{0}}\left(A_{p q}^{x} \frac{k_{0}^{2}-k_{y, q}^{2}}{k_{z, p q}}+A_{p q}^{y} \frac{k_{x, p} k_{y, q}}{k_{z, p q}}\right) .
\end{aligned}
$$

Once an expression for the fields in the gap region has been found, the field in each hole must be written too, in order to enforce the continuity of the tangential electric and magnetic fields among the two regions. Similar to 1D case, the suitable way is a modal expansion of rectangular-waveguide (RW) modes. Each hole in the lower surface can be regarded as a section of rectangular waveguide of length $h$ along the $z$ axis, connected to the gap region at $z=-g / 2$ and short circuited at the other side (at $z=-g / 2-h$ ). The electric and magnetic fields inside the hole, named here $\mathbf{E}^{\mathrm{WG}}$ and $\mathbf{H}^{\mathrm{WG}}$, respectively, can be expressed as a sum of rectangularwaveguide modes. Inside the hole on the plane $z=-g / 2$, the tangential electric field is

$$
\mathbf{E}_{\mathrm{t}}^{\mathrm{WG}}\left(x, y, z=-\frac{g}{2}\right)=\sum_{m n, i=e, h} r_{m n}^{-} C_{m n}^{i} \boldsymbol{\Phi}_{m n}^{i}(x, y)
$$

The superscripts ' $\mathrm{e}$ ' and ' $\mathrm{h}$ ' refer to transverse magnetic and transverse electric modes, respectively. The $C$ coefficients are the undetermined amplitudes of each mode, and the vector modal functions $\boldsymbol{\Phi}_{m n}^{h / e}$ are products of sinus and cosinus functions according to the well-known theory of rectangular waveguides [1]. Their expressions are given for completeness in the Appendix.

The magnetic field is

$$
\begin{gathered}
\eta_{0} \mathbf{H}_{\mathrm{t}}^{\mathrm{WG}}(z=-g / 2)= \\
\sum_{m n}\left[r_{m n}^{+} \frac{q_{z, m n}}{k_{0}} C_{m n}^{h}(-\hat{\mathbf{z}}) \times \boldsymbol{\Phi}_{m n}^{h}(x, y)\right. \\
\left.\quad+r_{m n}^{+} \frac{k_{0} \varepsilon_{\mathrm{r}}}{q_{z, m n}} C_{m n}^{e}(-\hat{\mathbf{z}}) \times \boldsymbol{\Phi}_{m n}^{e}(x, y)\right]
\end{gathered}
$$

where the transverse electric and magnetic modes appear in different terms due to their different admittances $\left(q_{m n} / k_{0} \eta_{0}\right.$ and $k_{0} \varepsilon_{\mathrm{r}} / q_{m n} \eta_{0}$, respectively).

In (30), $q_{z, m n}=\sqrt{\epsilon_{\mathrm{r}} k_{0}^{2}-m^{2} \pi^{2} / a^{2}-n^{2} \pi^{2} / a^{2}}$ is the wavenumber of the $(m, n)$ th mode $\left(\epsilon_{\mathrm{r}}\right.$ being the dielectric constant inside the hole), and

$$
r_{m n}^{ \pm}=1 \pm e^{-j 2 q_{z, m n} h}
$$

are, respectively, the magnetic-field and electric-field reflection coefficients due to the short-circuit at the end of the hole.

\section{E. Glide-symmetric boundary conditions for the $2 D$ structure}

With a way similar to the 1D case, in this subsection, by enforcing boundary conditions at the connection between the gap region and each hole, the dispersion equation of the structure is achieved. The boundary conditions will be formulated on the hole in the lower surface $(z=-g / 2,0<$ $x<a, 0<y<b)$ and on the hole in the upper surface $(z=g / 2, d / 2<x<a+d / 2, d / 2<y<b+d / 2)$. Moreover, in this structure, the generalized Floquet theorem discussed in [12] states that after a translation of half a period in both $x$ and $y$ directions and a mirroring around the glide plane $(z=0)$ the field is the same, apart a factor $\pm \exp \left(-j\left(k_{x, 0} d / 2+k_{y, 0} d / 2\right)\right)$, where the \pm sign depends on Floquet harmonic propagating in the structure (also in this case, we take the plus sign without loss of generality).

Now, to obtain the dispersion equation, first, we enforce the continuity of the electric field on the lower surface $(z=$ $-g / 2,0<x<a, 0<y<b)$ :

$$
\mathbf{E}_{\mathrm{t}}^{\mathrm{Gap}}(x, y, z=-g / 2)=\mathbf{E}_{\mathrm{t}}^{\mathrm{WG}}(x, y, z=-g / 2) .
$$

When the Floquet series (24) is replaced into (32) and inverted, we obtain

$$
\tilde{\mathbf{e}}_{\mathrm{t}, p q}^{\mathrm{Gap}}(z=-g / 2)=\tilde{\mathbf{E}}_{\mathrm{t}}^{\mathrm{WG}}\left(k_{x, p}, k_{y, q}\right),
$$

where

$$
\tilde{\mathbf{E}}_{\mathrm{t}}^{\mathrm{WG}}\left(k_{x, p}, k_{y, q}\right)=\sum_{m n, i=e, h} r_{m n}^{-} C_{m n}^{i} \tilde{\boldsymbol{\Phi}}_{m n}^{i}\left(k_{x, p}, k_{y, q}\right)
$$

is the Fourier transforms of $\mathbf{E}_{\mathrm{t}}^{\mathrm{WG}}(x, y, z=-g / 2)$ expressed in (29). Consequently, $\tilde{\boldsymbol{\Phi}}_{m n}^{i}\left(k_{x, p}, k_{y, q}\right)$ are the Fourier transforms of the modal functions $\boldsymbol{\Phi}$.

On the upper surface $(z=g / 2, d / 2<x<a+d / 2, d / 2<$ $y<b+d / 2$ ), by means of glide symmetry we can write

$$
\begin{aligned}
& \mathbf{E}_{\mathrm{t}}^{\mathrm{Gap}}(x, y, z=g / 2) \\
& =e^{-j\left(k_{x, 0} d+k_{y, 0} d\right) / 2} \mathbf{E}_{\mathrm{t}}^{\mathrm{WG}}\left(x-\frac{d}{2}, y-\frac{d}{2}, z=-\frac{g}{2}\right) .
\end{aligned}
$$

The Floquet series (24) is replaced into (35) and inverted by means of the Fourier-transform properties. Then, we obtain

$$
\tilde{\mathbf{e}}_{\mathrm{t}, p q}^{\mathrm{Gap}}(z=g / 2)=(-1)^{p+q} \tilde{\mathbf{E}}_{\mathrm{t}}^{\mathrm{WG}}\left(k_{x, p}, k_{y, q}\right) \text {. }
$$


If the two vector equations (33) and (36) are rewritten as four scalar equations, we can find an interesting symmetry property of Floquet harmonics in glide-symmetric structures:

$$
\begin{aligned}
-A_{p q}^{x} \sin \left(\theta_{z, p q}\right)+B_{p q}^{x} \cos \left(\theta_{z, p q}\right) & =\tilde{\mathbf{E}}_{\mathrm{t}}^{\mathrm{WG}} \\
A_{p q}^{x} \sin \left(\theta_{z, p q}\right)+B_{p q}^{x} \cos \left(\theta_{z, p q}\right) & =(-1)^{p+q} \tilde{\mathbf{E}}_{\mathrm{t}}^{\mathrm{WG}} \\
-A_{p q}^{y} \sin \left(\theta_{z, p q}\right)+B_{p q}^{y} \cos \left(\theta_{z, p q}\right) & =\tilde{\mathbf{E}}_{\mathrm{t}}^{\mathrm{WG}} \\
A_{p q}^{y} \sin \left(\theta_{z, p q}\right)+B_{p q}^{y} \cos \left(\theta_{z, p q}\right) & =(-1)^{p+q} \tilde{\mathbf{E}}_{\mathrm{t}}^{\mathrm{WG}}
\end{aligned}
$$

where $\theta_{z, p q}=k_{z, p q} g / 2$. The following final expressions for the $A$ and $B$ coefficients are obtained as functions of the modal-waveguide coefficients $C$.

If $p+q$ is even:

$$
\begin{aligned}
\left(\begin{array}{l}
A_{p q}^{x} \\
A_{p q}^{y}
\end{array}\right) & =\mathbf{0} \\
\left(\begin{array}{l}
B_{p q}^{x} \\
B_{p q}^{y}
\end{array}\right) & =\frac{\tilde{\mathbf{E}}_{\mathrm{t}}^{\mathrm{WG}}\left(k_{x, p}, k_{y, q}\right)}{\cos \left(\frac{k_{z, p q} g}{2}\right)}
\end{aligned}
$$

If $p+q$ is odd:

$$
\begin{aligned}
\left(\begin{array}{l}
A_{p q}^{x} \\
A_{p q}^{y}
\end{array}\right) & =-\frac{\tilde{\mathbf{E}}_{\mathrm{t}}^{\mathrm{WG}}\left(k_{x, p}, k_{y, q}\right)}{\sin \left(\frac{k_{z, p q} g}{2}\right)} \\
\left(\begin{array}{c}
B_{p q}^{x} \\
B_{p q}^{y}
\end{array}\right) & =\mathbf{0}
\end{aligned}
$$

By defining the parity of a $(p, q)$ harmonic as the parity of the number $p+q$, we can conclude that even (odd) harmonics have an even (odd) transverse electric field along the $z$ direction with respect to the plane $z=0$.

The coefficients $A$ and $B$ in (41) and (42) can now be replaced in the magnetic field expression (28). Since $A$ and $B$ are written in term of $C$, the coefficients $C$ are now the only quantities not yet determined. In order to solve the problem, we enforce the continuity of the tangential magnetic fields (25) and (30) across the hole aperture at $z=-g / 2$. Same as the 1D case, imposing the continuity condition on the upper surface $z=g / 2$ is not needed as it is automatically verified due to the parity properties (41) and (42) of the Floquet harmonics.

This continuity equation, not reported here for brevity, can be projected on scalar modal functions $\phi_{m^{\prime} n^{\prime}}^{x}(x, y)$ and $\phi_{m^{\prime} n^{\prime}}^{y}(x, y)$ (see Appendix) in order to obtain an algebraic linear system. For this purpose, we multiply both terms of the equation by $\phi_{m^{\prime} n^{\prime}}^{x}(x, y)$ and $\phi_{m^{\prime} n^{\prime}}^{y}(x, y)$ and integrate over the hole aperture. Using the orthonormal properties of scalar modal functions, the following linear system is then obtained:

$$
\left(\begin{array}{ll}
M_{m n}^{h, x} & M_{m n}^{e, x} \\
M_{m n}^{h, y} & M_{m n}^{e, y}
\end{array}\right) \cdot \mathbf{C}=\mathbf{0},
$$

where the relevant matrix elements are given by $(i=e, h)$

$$
\begin{aligned}
& M_{m n}^{i, x}=-\frac{r_{m n}^{-}}{j d^{2}} \sum_{p q}\left[\hat{\mathbf{x}} \cdot \tilde{\mathbf{\Phi}}_{m n}^{i} \tilde{\phi}_{m^{\prime} n^{\prime}}^{x} \frac{k_{0}^{2}-k_{y, q}^{2}}{k_{z, p q}} \tilde{f}_{p q}\right. \\
& \left.+\hat{\mathbf{y}} \cdot \tilde{\boldsymbol{\Phi}}_{m n}^{i} \tilde{\phi}_{m^{\prime} n^{\prime}}^{x} \frac{k_{x, p} k_{y, q}}{k_{z, p q}} \tilde{f}_{p q}\right]-\delta_{m m^{\prime}} \delta_{n n^{\prime}} Z_{m n}^{i, x} r_{m n}^{+}
\end{aligned}
$$

$$
\begin{aligned}
& M_{m n}^{i, y}=\frac{r_{m n}^{-}}{j d^{2}} \sum_{p q}\left[\hat{\mathbf{x}} \cdot \tilde{\boldsymbol{\Phi}}_{m n}^{i} \tilde{\phi}_{m^{\prime} n^{\prime}}^{y} \frac{k_{x, p} k_{y, q}}{k_{z, p q}} \tilde{f}_{p q}\right. \\
& \left.+\hat{\mathbf{y}} \cdot \tilde{\boldsymbol{\Phi}}_{m n}^{i} \tilde{\phi}_{m^{\prime} n^{\prime}}^{y} \frac{k_{0}^{2}-k_{x, p}^{2}}{k_{z, p q}} \tilde{f}_{p q}\right]-\delta_{m m^{\prime}} \delta_{n n^{\prime}} Z_{m n}^{i, y} r_{m n}^{+} .
\end{aligned}
$$

In these equations, we have suppressed the $\left(k_{x, p}, k_{y, q}\right)$ dependence of $\tilde{\boldsymbol{\Phi}}_{m n}^{e / h}$ and the $\left(-k_{x, p},-k_{y, q}\right)$ dependence of $\tilde{\phi}_{m^{\prime} n^{\prime}}^{x / y}$ for the sake of brevity. Moreover, the following equivalent impedances have been defined $Z_{m n}^{h, x}=m q_{z, m n}, Z_{m n}^{e, x}=$ $n k_{0}^{2} \varepsilon_{\mathrm{r}} / q_{z, m n}, Z_{m n}^{h, y}=n q_{z, m n}, Z_{m n}^{e, y}=m k_{0}^{2} \varepsilon_{\mathrm{r}} / q_{z, m n}$.

The vertical spectral function $f_{p q}$ is defined as

$$
\tilde{f}_{p q}=\left\{\begin{array}{ccc}
-\tan \left(\frac{k_{z, p q} g}{2}\right) & p+q & \text { even } \\
\cot \left(\frac{k_{z, p q} g}{2}\right) & p+q & \text { odd }
\end{array}\right.
$$

Note that this function defines the glide symmetry of a 2D periodic structure. Same as the 1D case, a different stratified $2 \mathrm{D}$ configuration (such as the unbounded air or a metal plate on the top of a single holey surface) would only require a different definition of $\tilde{f}_{p q}$.

It is interesting to write explicitly the expression of the dispersion relation obtained if only one rectangular-waveguide mode, whose modal function is $\Phi$, is retained in the hole expansion:

$$
\begin{aligned}
& \sum_{\substack{p+q \\
\text { even }}} \tilde{\Phi}\left(k_{x, p}, k_{y, q}\right) \tilde{\Phi}\left(-k_{x, p},-k_{y, q}\right) \frac{k_{0}^{2}-k_{y, q}^{2}}{k_{z, p q}} \cot \left(\frac{k_{z, p q} g}{2}\right) \\
- & \sum_{\substack{p+q \\
\text { odd }}} \tilde{\Phi}\left(k_{x, p}, k_{y, q}\right) \tilde{\Phi}\left(-k_{x, p},-k_{y, q}\right) \frac{k_{0}^{2}-k_{y, q}^{2}}{k_{z, p q}} \tan \left(\frac{k_{z, p q} g}{2}\right) \\
= & -j d^{2} \frac{r^{+}}{r^{-}} q_{z, 01} .
\end{aligned}
$$

The parity properties of different harmonics are clearly evident, if (47) is compared with the dispersion relation in the presence of a holey surface in air [52]. Furthermore, It is easy to realize that if $g \rightarrow+\infty$ and all $k_{z, p q}$ are imaginary (i.e., $k_{z, p q}=-j\left|k_{z, p q}\right|$ for bound modes), the above equations reduce to the holey surface in free space studied in [52].

\section{Numerical Results}

In this section, we perform dispersion analyses of glidesymmetric corrugated and holey surfaces with the modematching analysis presented in the previous section, implemented in Matlab. The analyses are compared for validation with analogous results obtained with a commercial software (CST Microwave Studio [46]).

\section{A. 1-D glide-symmetric corrugations: Effect of parameters variations}

In this subsection, the dispersion diagrams of glidesymmetric corrugated surfaces are computed for different values of all relevant physical and geometrical parameters. By testing the proposed method for different values of relevant parameters, not only we fully validate the numerical method presented here, but also we can investigate the effect of each parameter on the propagation characteristics of this structure. 
This investigation helps us to achieve a variation of the equivalent refractive index along the structure by smoothly varying these parameters across the surface. A specific structure is here used as a reference; the gap between the surfaces is $g=0.2$ $\mathrm{mm}$, the period is $p=4 \mathrm{~mm}$, the depth and the width of each groove is, respectively, $h=1.5 \mathrm{~mm}$ and $a=3 \mathrm{~mm}$, and the material filling each groove is air $\left(\varepsilon_{\mathrm{r}}=1\right)$.

In Fig. 3, the dispersion diagram of the reference glidesymmetric corrugation over the Brillouin zone is illustrated. This structure is invariant along $y$. However, to be able to compare the results of the mode matching method and $C S T$, periodic boundary conditions are enforced are assumed along $y$-direction, with a periodicity equal to $d / 2$. Thus, in this figure, a straight propagation along the $x$ direction is considered in the interval $\Gamma-\mathrm{X}\left(\beta_{y}=0\right)$, a propagation along the $y$ direction is considered in the interval $\mathrm{X}-\mathrm{M}\left(\beta_{x}=\pi / d\right.$ and $0<\beta_{y}<\pi /(d / 2)$. The sketch of the considered propagation constants is given in the inset of the figure. Comparing the propagation behavior of the glide-symmetric corrugation surfaces with the conventional corrugation, the absence of a stopband at the $\mathrm{X}$ point $(\beta d / \pi=1)$ of a glidesymmetric configuration is remarkable. This means that adding the glide symmetry to a periodic structure makes the first mode of the structure less dispersive.

Fig. 4 shows the effect of the variation of the material filling each groove on propagation characteristics. Three values are selected: $\varepsilon_{\mathrm{r}}=1, \varepsilon_{\mathrm{r}}=2, \varepsilon_{\mathrm{r}}=3$. A perfect agreement between the mode-matching code and CST is visible in this figure. As expected, the equivalent refractive index is higher for denser materials filling the grooves. However, achieving a spatial variation of this refractive index by filling the grooves of the structure with different material is not easy in fabrication. Thus, this result is considered here mainly for the code validation, and no specific commercial materials have been further considered.

Fig. 5 and 6, respectively, demonstrate the dispersion diagrams for different values of the width and the depth of

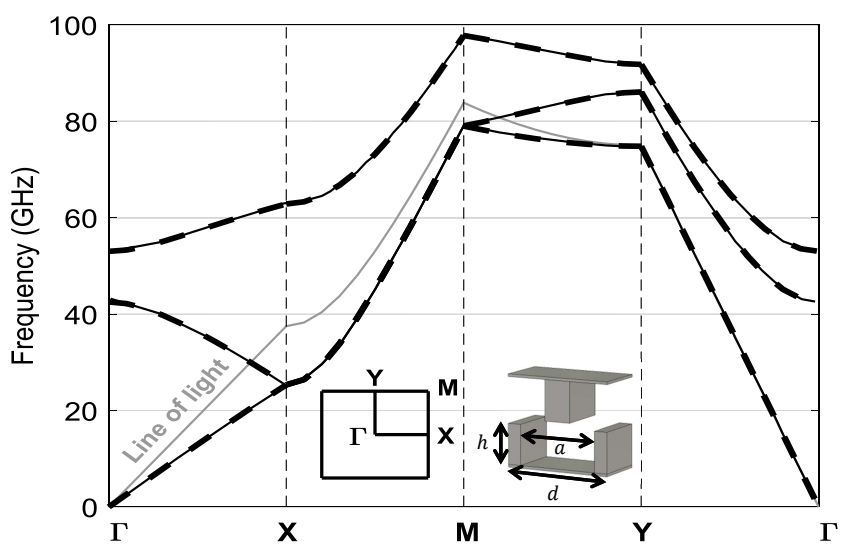

Fig. 3. Dispersion diagram of the glide-symmetric corrugated surfaces illustrated in Fig. 2. Geometric parameters: $d=4 \mathrm{~mm}, a=3 \mathrm{~mm}, g=0.2$ $\mathrm{mm}, h=1.5 \mathrm{~mm}$. Mode matching presented in this paper (solid line), CST (dashed line), line of light (gray line). the grooves. Three values are tested for the width of the grooves: $a=3 \mathrm{~mm}, a=2.5 \mathrm{~mm}, a=2 \mathrm{~mm}$. And three values are tested for the height of the grooves: $h=3 \mathrm{~mm}$, $h=2.5 \mathrm{~mm}, h=2 \mathrm{~mm}$. The results show the equivalent refractive index increases if the depth of the grooves increases or the width of the grooves decreases. Note that after a certain depth, increasing the depth does not have effect on the dispersion diagram anymore since all PPW modes in the grooves, which were described in Subsection II-B, will be exponentially attenuating due to the subwavelength width of the grooves, and the fields does not penetrate deep inside the grooves. As a result, after a certain threshold, a variation of the depth of the corrugation does not change significantly the field distribution in the structure.

Finally, it should be mentioned that all the results are obtained by considering only five PPW modes inside the

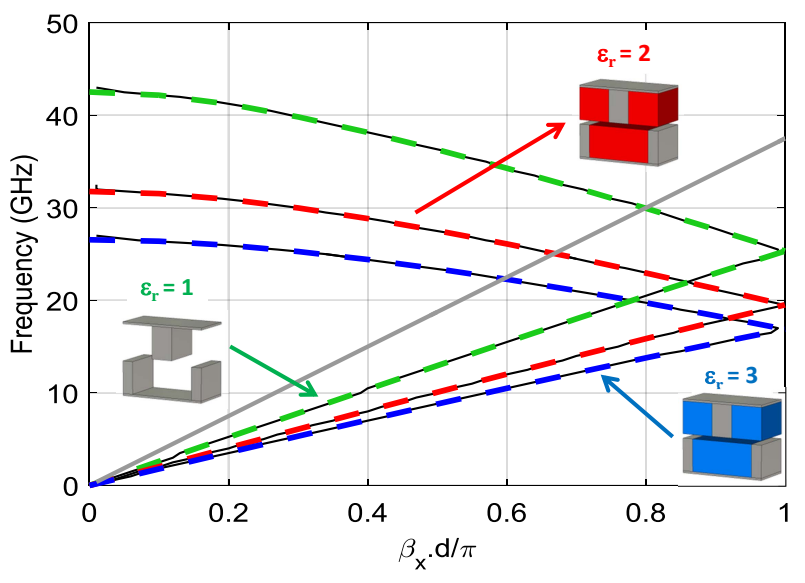

Fig. 4. Dispersion diagram of the glide-symmetric corrugated surfaces illustrated in Fig. 2. Geometric parameters: $d=4 \mathrm{~mm}, a=3 \mathrm{~mm}, g=0.2$ $\mathrm{mm}, h=1.5 \mathrm{~mm}$. The grooves are filled with different dielectrics. Mode matching presented in this paper (solid lines), CST (dashed lines), line of light (gray line).

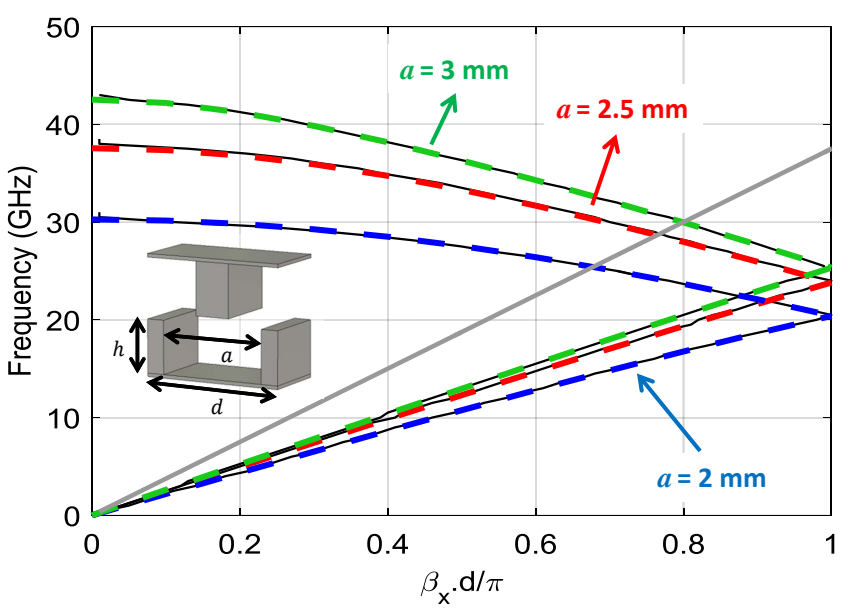

Fig. 5. Dispersion diagram of the glide-symmetric corrugated surfaces illustrated in Fig. 2. Geometric parameters: $d=4 \mathrm{~mm}, g=0.2 \mathrm{~mm}, h=1.5$ $\mathrm{mm}$. Mode matching presented in this paper (solid lines), CST (dashed lines), line of light (gray line). 
grooves $(M=4)$ and seven Floquet modes in the gap region $(P=3)$ except the case that smaller values of $a$ are studied, in which five PPW modes and eleven Floquet modes were taken into account. As it was explained in Subsection II-C, for smaller values of the ratio $a / d$, the ratio of the PPW modes to Floquet modes should be smaller.

\section{B. 2-D glide-symmetric holes: Effect of parameter variations}

In this subsection, the dispersion diagram of 2-D holey structures are computed to study the influence of the variation of all relevant physical and geometrical parameters. The results are computed with the mode-matching and with CST. A variation of the physical parameters leads to achieve a variation of the equivalent refractive index along the waveguide by smoothly varying these parameters across the surface, and assuming a local periodicity of the resulting lattice. Here, we employ as a reference a structure with a gap between the

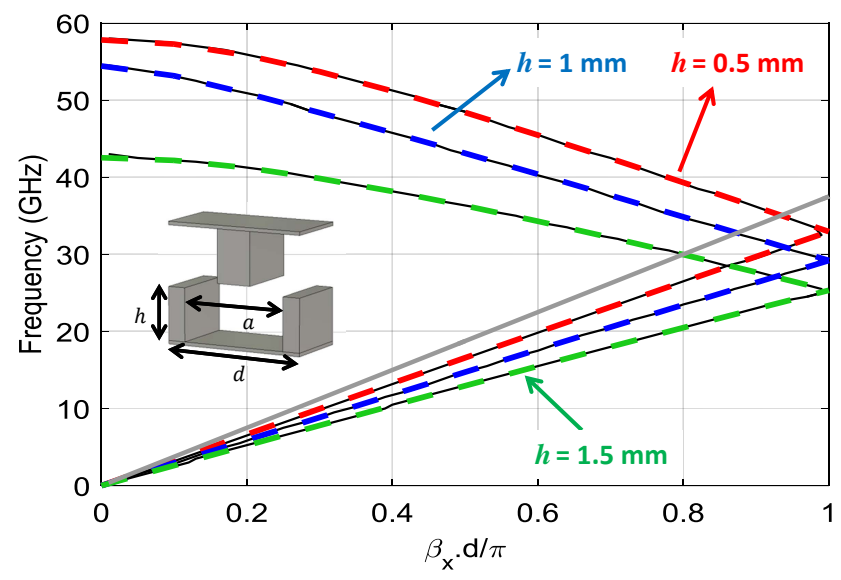

Fig. 6. Dispersion diagram of the glide symmetric corrugated surfaces illustrated in Fig. 2. Geometric parameters: $d=4 \mathrm{~mm}, a=3 \mathrm{~mm}, g=0.2$ $\mathrm{mm}$. Mode matching presented in this paper (solid lines), CST (dashed lines), line of light (gray line)

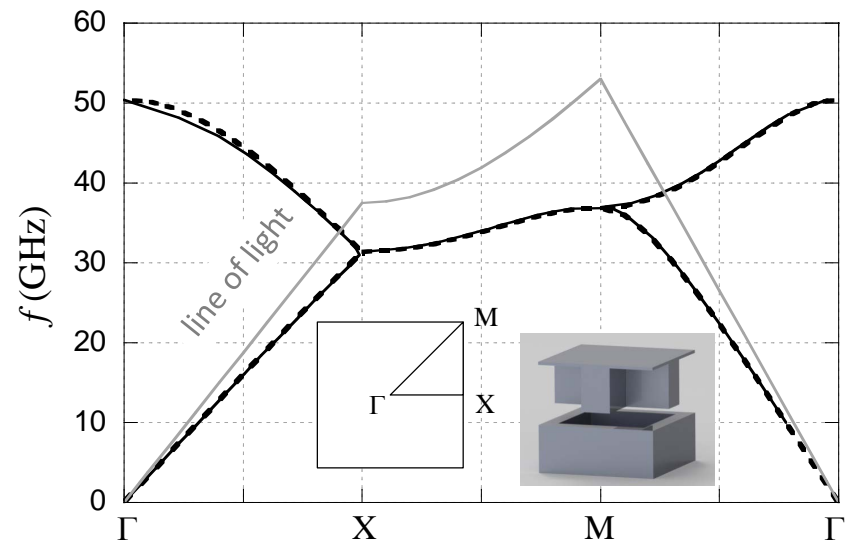

Fig. 7. Dispersion diagram of the glide-symmetric unit cell with squared hole in Fig. 1(b). Geometric parameters: $d=4 \mathrm{~mm}, a=b=3 \mathrm{~mm}, g=0.5$ $\mathrm{mm}, h=1.5 \mathrm{~mm}$. Mode matching presented in this paper (solid line), CST (dashed line), line of light (gray line). surfaces $g=0.5 \mathrm{~mm}$, period $p=4 \mathrm{~mm}$, depth of hole $h=1.5$ $\mathrm{mm}$, lateral size of the hole $a=3 \mathrm{~mm}$, and the material filling the hole is air $\left(\varepsilon_{\mathrm{r}}=1\right)$.

For completeness, the dispersion diagram of the reference glide-symmetric structure over the irreducible Brillouin zone is shown in Fig. 7. Together with the glide-symmetric case, the one-sided holey structure is also analyzed in order to highlight the different modal behaviour of the structures. In this figure, a straight propagation along the $x$ direction is considered in the interval $\Gamma-\mathrm{X}\left(\beta_{y}=0\right)$, a propagation along the $y$ direction is considered in the interval $\mathrm{X}-\mathrm{M}\left(\beta_{x}=\pi / d, \beta_{y}=0\right)$, and a skewed propagation is considered in the interval $\mathrm{M}-\Gamma\left(\beta_{x}=\right.$ $\left.\beta_{y} \neq 0\right)$. The comparison with CST validates the method in this most general case. A one-sided structure was analyzed with a mode-matching developed ah-hoc in [43]. In Fig. 7, the equivalent refractive index is computed for both the glidesymmetric and the one-sided structure, both in the straight and in the skewed propagation. Note that the index is substantially the same, which proves an excellent isotropy of the glidesymmetric configuration. It is especially important to remark the absence of a stopband at the $\mathrm{X}$ point $\beta_{x} d / \pi=1$, which is a characteristic feature of glide-symmetric configurations, in contrast with the usual stopband behavior of the one-sided structure.

In Fig. 8, the variation of the material filling each hole is studied. Three values are selected: $\varepsilon_{\mathrm{r}}=1, \varepsilon_{\mathrm{r}}=2, \varepsilon_{\mathrm{r}}=3$. As shown in the figure, a perfect agreement is obtained between the mode-matching code and CST. As expected, the equivalent refractive index is higher for denser materials filling the holes. However, achieving a spatial variation of this refractive index by filling the holes of the structure with different materials is not easy to be manufactured in microwave regimes. For this reason, this result is considered here mainly for the code validation, and no specific commercial materials have been further considered.

In Fig. 9, the variation of the gap between surfaces is

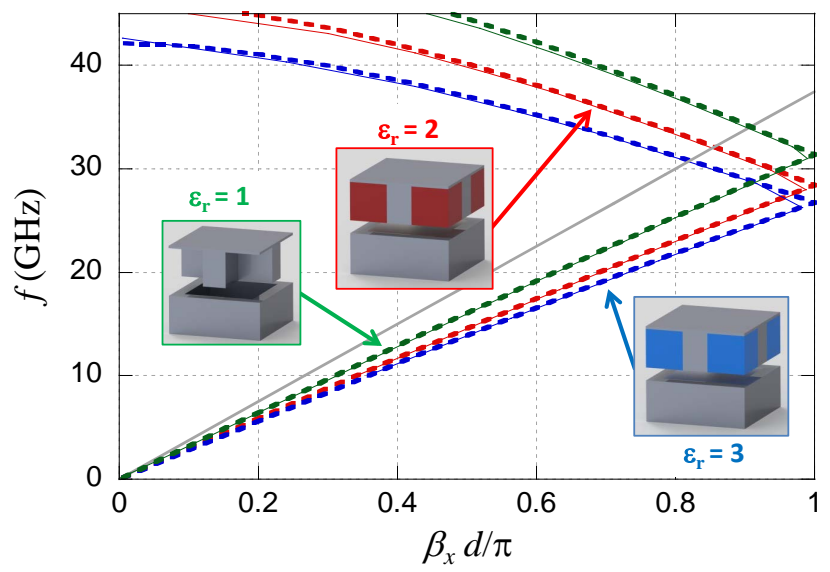

Fig. 8. Dispersion diagram of the glide-symmetric unit cell with squared hole in Fig. 1(b). Geometric parameters: $d=4 \mathrm{~mm}, a=b=3 \mathrm{~mm}, g=0.5 \mathrm{~mm}$, $h=1.5 \mathrm{~mm}$. The holes are filled with different dielectrics. Mode matching presented in this paper (solid lines), CST (dashed lines), line of light (gray line). 
studied. Three values are selected: $g=0.5 \mathrm{~mm}, g=0.3$ $\mathrm{mm}, g=0.2 \mathrm{~mm}$. A small discrepancy between CST and the mode matching appears for extremely small values of $g$ such as $0.2 \mathrm{~mm}$. However, in this extreme case an accurate solution of CST is not granted either, due to the difficult meshing of the small air region between the surfaces. Furthermore, the refractive indexes obtained with the two methods are close enough that the practical implementation of a lens would not reveal a significant difference. The equivalent refractive index increases as the thickness of the gap increases, due to stronger interaction between the surfaces. However, also in this case varying the gap in order to obtain a spatial variation of the index is not practical.

In Fig. 10, the variation of the lateral size of the squared hole is studied. Three values are selected: $a=3 \mathrm{~mm}, a=2.5$ $\mathrm{mm}, a=2 \mathrm{~mm}$. As shown in the figure, a perfect agreement is obtained between the mode-matching code and CST. As expected, as the size of the hole becomes smaller, propagation

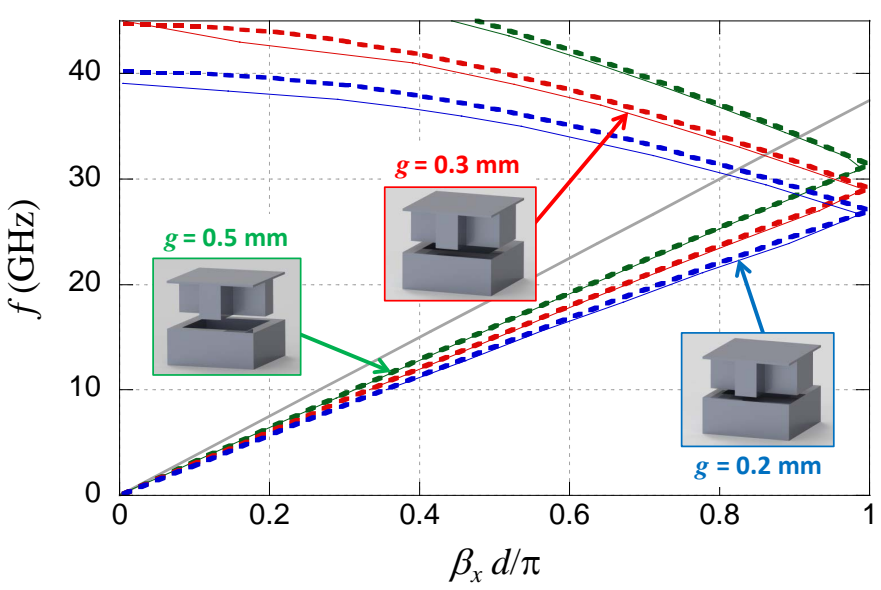

Fig. 9. Dispersion diagram of the glide-symmetric unit cell with squared hole in Fig. 1(b). Geometric parameters: $d=4 \mathrm{~mm}, a=b=3 \mathrm{~mm}, h=1.5$ $\mathrm{mm}$. Mode matching presented in this paper (solid lines), CST (dashed lines), line of light (gray line).

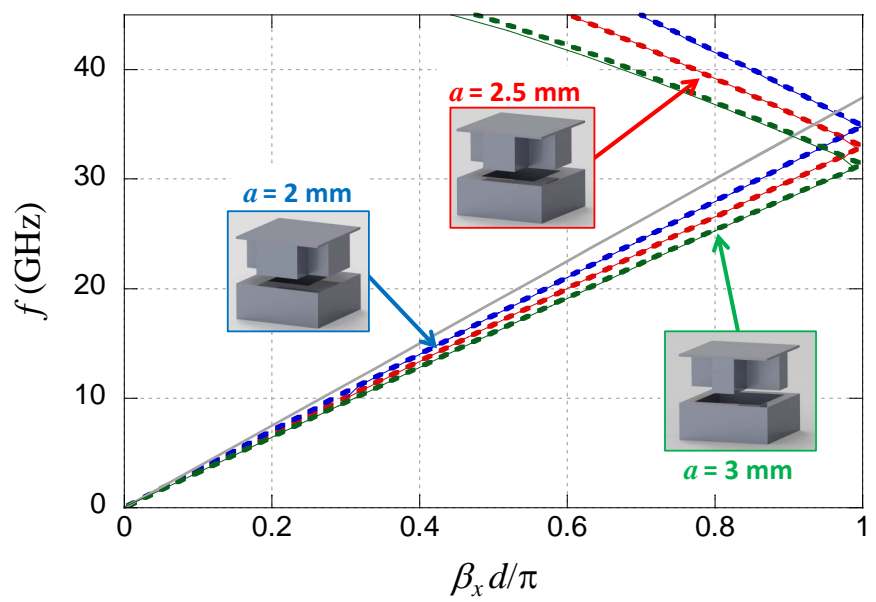

Fig. 10. Dispersion diagram of the glide-symmetric unit cell with squared hole in Fig. 1(b). Geometric parameters: $d=4 \mathrm{~mm}, a=b, g=0.5 \mathrm{~mm}$, $h=1.5 \mathrm{~mm}$. Mode matching presented in this paper (solid lines), CST (dashed lines), line of light (gray line). tends to approach the free-space limit.

Finally, in Fig. 11, the variation of the depth of holes is studied. Three values are selected: $h=3 \mathrm{~mm}, h=2.5 \mathrm{~mm}$, $h=2 \mathrm{~mm}$. Again, the perfect agreement of the methods fully validates the mode matching. As the depth of the holes increases, the equivalent refractive index increases too. It is interesting to note that this increase occurs up to a certain depth, depending on the size of the hole and on the frequency. In fact, as explained in the previous section, the field in each hole can be regarded as a superposition of rectangularwaveguide modes. Due to the subwavelength size of each hole, all these modes will be exponentially attenuating, so that the field does not penetrate deep inside the hole. After a certain threshold, a variation of the depth of the hole does not change significantly the field distribution in the structure.

These last two analyses are the most important for practical implementations since it is relatively simple to drill holes of given lateral dimensions and depth, so that a tapering of the refractive index can be achieved along the surface by smoothly varying these two parameters. The refractive index variation range is sufficient to implement well-known lenses such as the Luneburg one, as already demonstrated in [14].

The mode matching method is much faster than CST eigenmode analyses. This is due to the fact that CST needs to mesh the whole volume of the periodic cell, while the mode matching has unknowns only on one hole of the cell. It is interesting to remark that the second glide-symmetric hole does not require additional degrees of freedom, thanks to the enforced symmetry condition. The number of RW modes retained in the calculation depends on the parameters of the structure. However, in all the structures of interest for artificial lenses the first low-order mode (e.g., the one going from 0 to $30 \mathrm{GHz}$ in Fig. 7) is always correctly tracked with the choice $n=1,2,3$ and $m=1, \cdots, 4$ in (29). This choice leads to a mode-matching computation time for a single frequency point of $0.7 \mathrm{~s}$, compared with a CST simulation time of $47 \mathrm{~s}$.

At higher frequencies, the higher-order modes (e.g., from

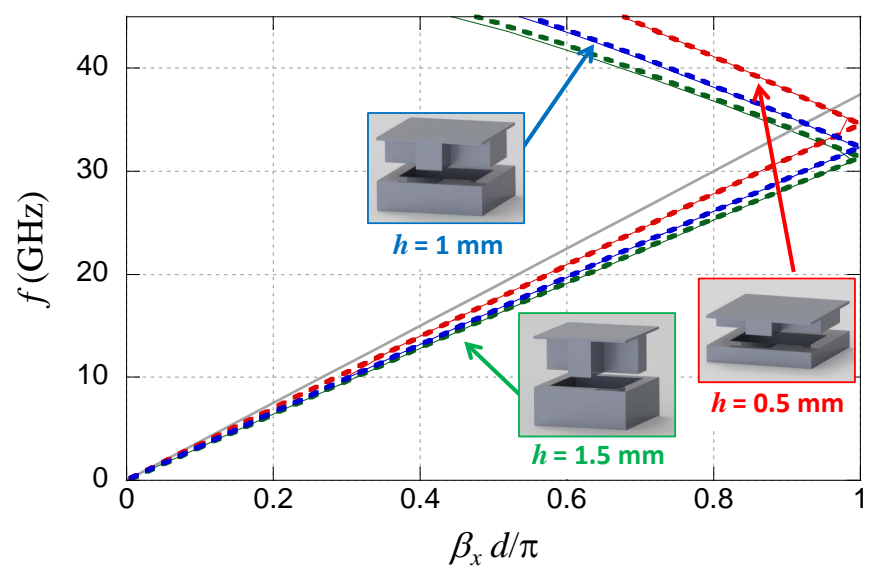

Fig. 11. Dispersion diagram of the glide symmetric unit cell with squared hole in Fig. 1(b). Geometric parameters: $d=4 \mathrm{~mm}, a=b=3 \mathrm{~mm}, g=0.5$ $\mathrm{mm}$. Mode matching presented in this paper (solid lines), CST (dashed lines), line of light (gray line). 
30 to $50 \mathrm{GHz}$ in Fig. 7) are more sensitive to the value of the gap $g$ : as $g$ decreases by keeping a fixed period, more RW modes are necessary to describe the high-frequency field in the holes, thus slowing down the calculation. However, a similar effect can be observed in CST, due to the need to accurately mesh a very thin gap. It was observed that the high-frequency analysis for the extreme case of $g=0.2 \mathrm{~mm}$ (Fig. 8) required $n=1, \cdots, 7$ and $m=1, \cdots, 9$, resulting in a computation time of $17.8 \mathrm{~s}$ per frequency with respect to a $C S T$ computation of $272 \mathrm{~s}$.

All the tests have been performed on an Intel Xeon CPU X5650@2.67 GHz with $96 \mathrm{GHz}$ of RAM. The computation time of the mode matching could be further reduced by implementing a zero-finding algorithm to locate the zeros of the determinant of (43) [53]. In the present computations, the zeros have been located by simply taking the minimum of the magnitude of the determinant on a real segment (e.g., $\beta_{x} d / \pi$ in $[0,1], \beta_{y}=0$, and $\alpha_{x}=\alpha_{y}=0$ for a propagation along the $x$ direction).

\section{2-D glide-symmetric holes: Anisotropy}

In this subsection, we study glide-symmetric structures having rectangular holes rather than squared ones. As expected, this asymmetry along the two periodicity axis leads to a certain degree of anisotropy, which is a promising result to realize lenses requiring anisotropic materials.

In Fig. 1(c), the unit cell of a glide-symmetric structure with rectangular holes has been shown. Two different cases whose lateral dimensions are $a=3 \mathrm{~mm}, b=a / 2=1.5 \mathrm{~mm}$ and $a=3 \mathrm{~mm}, b=a / 4=0.75 \mathrm{~mm}$ have been studied. Moreover, their results are compared with a case having squared hole (Fig. 1(b)) with lateral dimensions $a=b=3 \mathrm{~mm}$ as the reference case.

In Fig. 12 the full dispersion diagrams of the structures with rectangular holes, as well as, the comparison of their dispersion diagrams and the dispersion diagram of the reference case are shown. Propagation along the $y$ direction is mainly affected by the value of $a$ (length of hole along $x$ direction) since the three curves are superimposed and the variation of $b$ (length of hole along $y$ direction) does not affect the dispersion. On the other hand, propagation along the $x$ direction is rather related to $b$ : if $b$ becomes small, the dispersion diagram approaches the line of light as commented in Fig. 12.

Most interestingly, the curves obtained in the intervals $\Gamma-\mathrm{X}$ and $\mathrm{Y}-\Gamma$ are extremely straight. This means that the refractive index of the Bloch mode is constant over a ultralarge frequency band, along both the propagation direction. This is illustrated in Fig. 13, where the equivalent refractive indexes are shown as a function of frequency for the three cells. The refractive indexes referring to propagation along the $x$ axis are compared with those referring to propagation along the $y$ axis. As already said, a narrow hole leads allows one to synthesize a desired effective refractive index along one direction by keeping an index almost equal to 1 in the orthogonal direction. The lack of frequency dispersion is also visible in the figure.

This kind of behavior can pave the way for the first time to the realization of all-metal flat UWB anisotropic lenses, thus promising a great flexibility in the synthesis of aperture fields

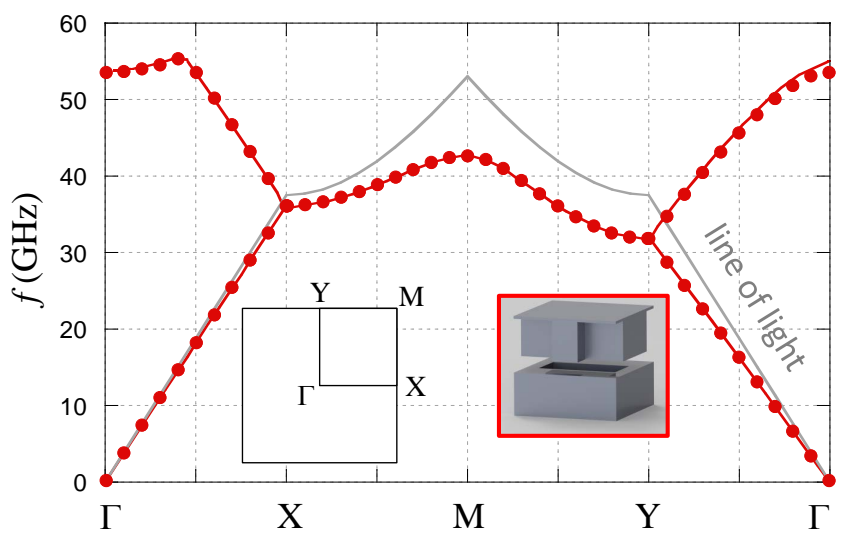

(a)

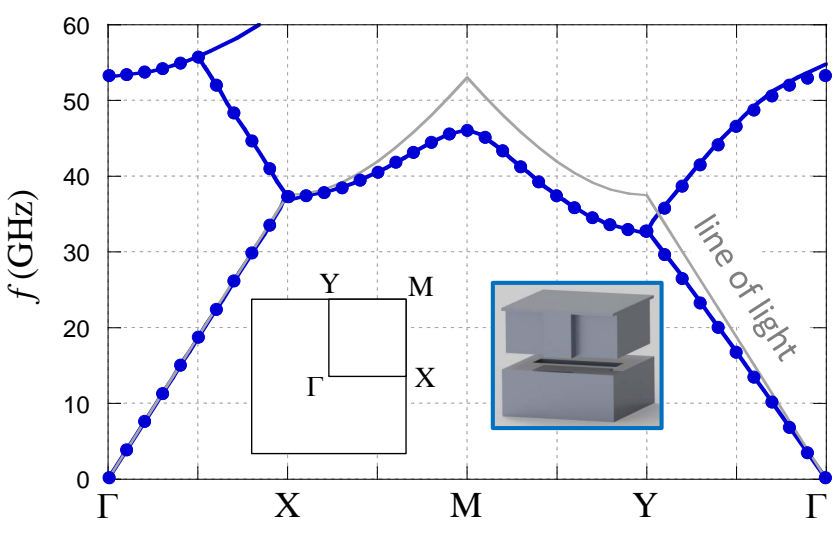

(b)

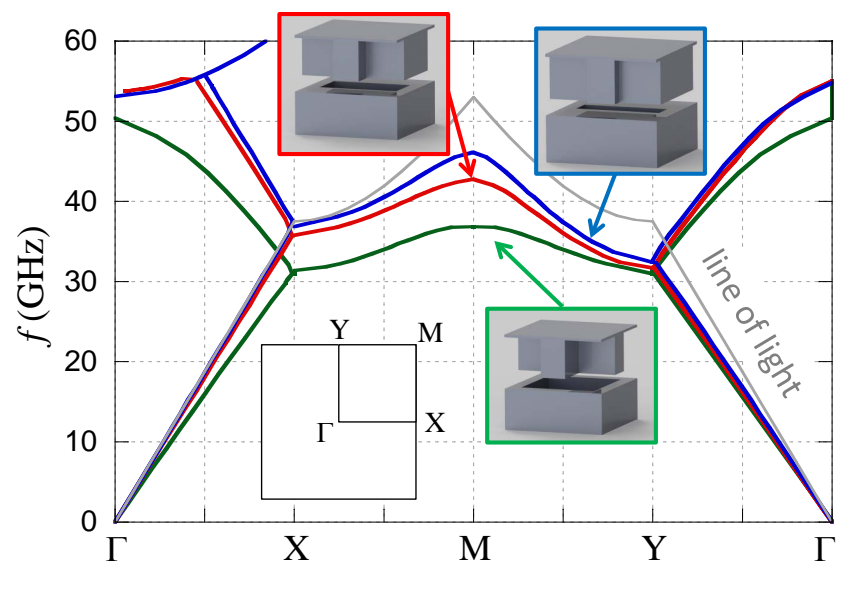

(c)

Fig. 12. Dispersion diagram of the glide-symmetric unit cell with square and rectangular hole in Fig. 1(b)-(c). Geometric parameters: $d=4 \mathrm{~mm}, a=3$ $\mathrm{mm} g=0.5 \mathrm{~mm}, h=1.5 \mathrm{~mm}$. Mode matching presented in this paper (solid lines), CST (dots), line of light (gray line). (a) $b=a / 2=1.5 \mathrm{~mm}$, (b) $b=a / 4=0.75 \mathrm{~mm}$, (c) comparison among the squared case $(a=b=4$ $\mathrm{mm}$, green line), and the rectangular cases $b=a / 2=1.5$ (red line) $\mathrm{mm}$ and $b=a / 4=0.75 \mathrm{~mm}$ (blue line). 
or antenna shape (e.g., if transformation optics is used for the lens design [54],[55]).

\section{Conclusion}

A mode matching method for the dispersion analysis of glide-symmetric corrugated and holey surfaces are presented. Using the higher symmetry of the structure and the generalized Floquet theorem, the imposed boundary conditions are simplified compared to a periodic structure without higher symmetry. We have also shown an interesting symmetry that exists in the Floquet harmonics propagating in glide-symmetric structures.

The method has been validated by comparing the results of the presented method for several cases with the results of the commercial software CST. The effect of variation of different parameters in the propagation characteristics of glidesymmetric corrugated and holey structures with both square and rectangular holes has been studied. Moreover, the full dispersion diagrams of these structures have been obtained to verify the accuracy of the method for propagation in all direction. In all test cases, a good agreement between the results of the mode matching method and the commercial software CST is remarkable.

This study confirmed the interesting characteristics of glidesymmetric structures, i.e. the absence of the band gap at the point $\mathrm{X}$ of the Brillouin zone and a low frequency dispersion on a wide range of frequencies. Thus, the proposed method, which is much faster than the commercial software, can be employed for designing all-metal glide-symmetric metasurfaces with UWB isotropic and anisotropic features. These metasurfaces are appropriate candidates for the fabrication of low-cost and low-loss graded-index planar lenses and EBG structures.

\section{APPENDIX}

\section{MODAL FUNCTIONS OF RECTANGULAR WAVEGUIDES}

According to the theory of rectangular waveguides, the modal functions $\boldsymbol{\Phi}_{m n}^{h / e}$ for a waveguide with square cross

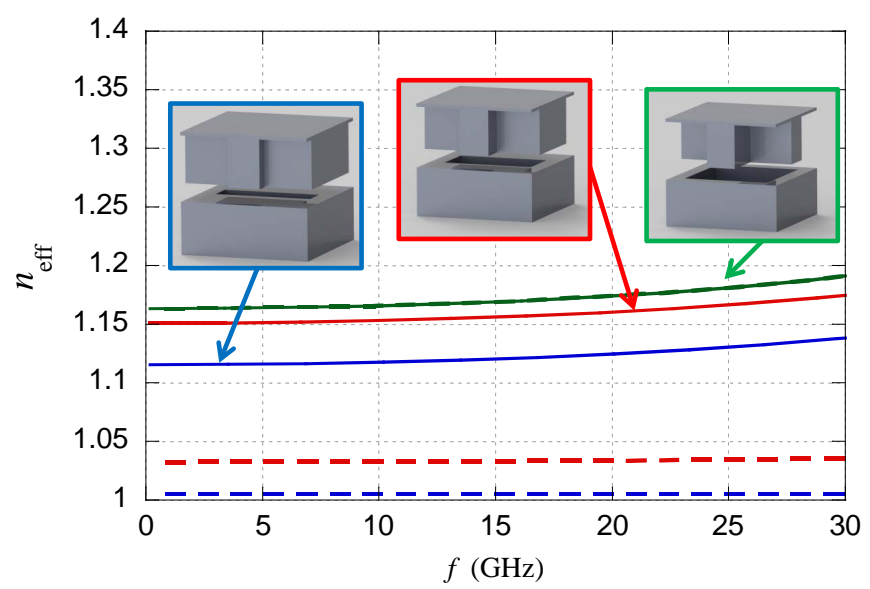

Fig. 13. Effective refractive index of the glide-symmetric unit cell with square and rectangular hole in Fig. 7 (green lines), Fig. 1(b) (red lines), and 1(c) (blue lines). Propagation along the $x$ axis (dashed lines) and the $y$ axis (solid lines). section of length $a$ can be expressed as

$$
\begin{aligned}
& \boldsymbol{\Phi}_{m n}^{h}(x, y)=\hat{\mathbf{x}} n \phi_{m n}^{x}(x, y)-\hat{\mathbf{y}} m \phi_{m n}^{y}(x, y), \\
& \boldsymbol{\Phi}_{m n}^{e}(x, y)=\hat{\mathbf{x}} m \phi_{m n}^{x}(x, y)+\hat{\mathbf{y}} n \phi_{m n}^{y}(x, y),
\end{aligned}
$$

where we have defined the orthonormal scalar modal functions as

$$
\phi_{m n}^{x}(x, y)=\left\{\begin{array}{cc}
\sqrt{\frac{2}{a}} \cos \left(\frac{m \pi x}{a}\right) \sqrt{\frac{2}{a}} \sin \left(\frac{n \pi y}{a}\right) & m \neq 0 \\
\sqrt{\frac{1}{a}} \sqrt{\frac{2}{a}} \sin \left(\frac{n \pi y}{a}\right) & m=0
\end{array}\right.
$$

and $\phi_{m n}^{y}(x, y)=\phi_{n m}^{x}(y, x)$.

In the case of a rectangular waveguide with the length of $a$ and the width of $b$, the modal functions $\boldsymbol{\Phi}_{m n}^{h / e}$ are

$$
\begin{aligned}
& \boldsymbol{\Phi}_{m n}^{h}(x, y)=\hat{\mathbf{x}} n \phi_{m n}^{x}(x, y)-\hat{\mathbf{y}} m \phi_{m n}^{y}(x, y), \\
& \boldsymbol{\Phi}_{m n}^{e}(x, y)=\hat{\mathbf{x}} m \phi_{m n}^{x}(x, y)+\hat{\mathbf{y}} n \phi_{m n}^{y}(x, y),
\end{aligned}
$$

where we have defined the orthonormal scalar modal functions as

$$
\phi_{m n}^{x}(x, y)=\left\{\begin{array}{cc}
\sqrt{\frac{2}{a}} \cos \left(\frac{m \pi x}{a}\right) \sqrt{\frac{2}{b}} \sin \left(\frac{n \pi y}{b}\right) & m \neq 0 \\
\sqrt{\frac{1}{a}} \sqrt{\frac{2}{b}} \sin \left(\frac{n \pi y}{b}\right) & m=0
\end{array}\right.
$$

and

$$
\phi_{m n}^{y}(x, y)=\left\{\begin{array}{cc}
\sqrt{\frac{2}{a}} \sin \left(\frac{m \pi x}{a}\right) \sqrt{\frac{2}{b}} \cos \left(\frac{n \pi y}{b}\right) & n \neq 0 \\
\sqrt{\frac{2}{a}} \sin \left(\frac{m \pi x}{a}\right) \sqrt{\frac{1}{b}} & n=0
\end{array} .\right.
$$

\section{REFERENCES}

[1] D. M. Pozar, "Microwave Engineering." John Wiley and Sons, Inc., 2011, ch. 8: Microwave Filters.

[2] J. D. Joannopoulos and S. G. Johnson, Photonic Crystals: Molding the Flow of Light. Princeton University Press, 2008.

[3] G. V. Eleftheriades and K. G. Balmain, Negative-Refraction Metamaterials: Fundamental Principles and Applications. Wileyy-IEEE Press, 2005.

[4] R. Collin and F. Zucker, Antenna Theory, ser. Inter-University Electronics Series. McGraw-Hill, 1969, no. pt. 2

[5] J. Volakis, Antenna Engineering Handbook, Fourth Edition. McGraw-Hill Companies,Incorporated, 2007. [Online]. Available: https://books.google.it/books?id=bmdFAAAAYAAJ

[6] Z. Wang, Y. Chong, J. D. Joannopoulos, and M. Soljacic, "Observation of unidirectional backscattering-immune topological electromagnetic states," Nature, vol. 461, pp. 772-775, Oct 2009. [Online]. Available: https://link.aps.org/doi/10.1103/PhysRevLett.100.013904

[7] A. B. Khanikaev, S. H. Mousavi, W.-K. Tse, M. Kargarian, A. H. MacDonald, and S. G., "Photonic topological insulators," Nature Materials, vol. 233, pp. 772-775, Oct 2013. [Online]. Available: https://link.aps.org/doi/10.1103/PhysRevLett.100.013904

[8] A. Figotin and I. Vitebskiy, "Gigantic transmission bandedge resonance in periodic stacks of anisotropic layers," Phys. Rev. E, vol. 72, p. 036619, Sep 2005. [Online]. Available: https://link.aps.org/doi/10.1103/PhysRevE.72.036619

[9] M. A. K. Othman, X. Pan, G. Atmatzakis, C. G. Christodoulou, and F. Capolino, "Experimental demonstration of degenerate band edge in metallic periodically loaded circular waveguide," IEEE Transactions on Microwave Theory and Techniques, vol. PP, no. 99, pp. 1-9, 2017.

[10] A. D. Sabata, L. Matekovits, and O. Lipan, "Band pattern of commensurate modulated periodic structures," IET Microwaves, Antennas Propagation, vol. 11, no. 9, pp. 1303-1307, 2017.

[11] F. D. M. Haldane and S. Raghu, "Possible realization of directiona optical waveguides in photonic crystals with broken time-reversal symmetry," Phys. Rev. Lett., vol. 100, p. 013904, Jan 2008. [Online]. Available: https://link.aps.org/doi/10.1103/PhysRevLett.100.013904

[12] A. Hessel, M. H. Chen, R. C. M. Li, and A. A. Oliner, "Propagation in periodically loaded waveguides with higher symmetries," Proceedings of the IEEE, vol. 61, no. 2, pp. 183-195, Feb. 1973. 
[13] O. Dahlberg, R. C. Mitchell-Thomas, and O. Quevedo-Teruel, "Reducing the dispersion of periodic structures with twist and polar glide symmetries," Scientific Reports, vol. 7, no. 10136, 2017.

[14] O. Quevedo-Teruel, M. Ebrahimpouri, and M. N. M. Kehn, "Ultrawideband metasurface lenses based on off-shifted opposite layers," IEEE Antennas and Wireless Propagation Letters, vol. 15, pp. 484-487, Dec. 2016.

[15] M. Ebrahimpouri, E. Rajo-Iglesias, Z. Sipus, and O. Quevedo-Teruel, "Cost-effective gap waveguide technology based on glide-symmetric holey ebg structures," IEEE Transactions on Microwave Theory and Techniques, vol. 66, no. 2, pp. 927-934, Feb 2018.

[16] S. Sensiper, "Electromagnetic wave propagation on helical structures (a review and survey of recent progress)," Proceedings of the IRE, vol. 43, no. 2, pp. 149-161, Feb 1955.

[17] P. J. Crepeau and P. R. McIsaac, "Consequences of symmetry in periodic structures," Proceedings of the IEEE, vol. 52, no. 1, pp. 33-43, Jan 1964

[18] R. Mittra and S. Laxpati, "Propagation in a wave guide with glide reflection symmetry," Canadian Journal of Physics, vol. 43, no. 2, pp. 353-372, 1965. [Online]. Available: https://doi.org/10.1139/p65-032

[19] R. Kieburtz and J. Impagliazzo, "Multimode propagation on radiating traveling-wave structures with glide-symmetric excitation," IEEE Transactions on Antennas and Propagation, vol. 18, no. 1, pp. 3-7, Jan 1970.

[20] R. Quesada, D. Martín-Cano, F. J. García-Vidal, and J. Bravo-Abad, "Deep-subwavelength negative-index waveguiding enabled by coupled conformal surface plasmons," Opt. Lett., vol. 39, no. 10, pp. 2990-2993, May 2014.

[21] J. J. Wu, C.-J. Wu, D. J. Hou, K. Liu, and T.-J. Yang, "Propagation of low-frequency spoof surface plasmon polaritons in a bilateral crossmetal diaphragm channel waveguide in the absence of bandgap," IEEE Photonics Journal, vol. 7, no. 1, pp. 1-8, Feb. 2015

[22] D. Cavallo and C. Felita, "Analytical formulas for artificial dielectrics with non-aligned layers," IEEE Transactions on Antennas and Propagation, vol. PP, no. 99, pp. 1-1, 2017.

[23] T. Chang, J. U. Kim, S. K. Kang, H. Kim, D. K. Kim, Y.-H. Lee, and J. Shin, "Broadband giant-refractive-index material based on mesoscopic space-filling curves," Nature Materials, vol. 7, pp. 772-775, Aug 2016. [Online]. Available: https://www.nature.com/articles/ncomms12661

[24] J. Miao, "Ka-band 2d luneburg lens design with glidesymmetric metasurface," Master's thesis, KTH, Electromagnetic Engineering, 2017. [Online]. Available: http://www.divaportal.org/smash/record.jsf?pid=diva2\%3A1142083\&dswid=1831

[25] C. Walter, "Surface-wave Luneberg lens antennas," IRE Transactions on Antennas and Propagation, vol. 8, no. 5, pp. 508-515, Sep. 1960.

[26] S. Maci, G. Minatti, M. Casaletti, and M. Bosiljevac, "Metasurfing: Addressing waves on impenetrable metasurfaces," IEEE Antennas and Wireless Propagation Letters, vol. 10, pp. 1499-1502, 2011.

[27] M. Bosiljevac, M. Casaletti, F. Caminita, Z. Sipus, and S. Maci, "Nonuniform metasurface Luneburg lens antenna design," IEEE Transactions on Antennas and Propagation, vol. 60, no. 9, pp. 4065-4073, Sep. 2012.

[28] A. Grbic and G. V. Eleftheriades, "Periodic analysis of a 2-D negative refractive index transmission line structure," IEEE Transactions on Antennas and Propagation, vol. 51, no. 10, pp. 2604-2611, Oct. 2003.

[29] C. Pfeiffer and A. Grbic, "A printed, broadband Luneburg lens antenna," IEEE Transactions on Antennas and Propagation, vol. 58, no. 9, pp. 3055-3059, Sep. 2010.

[30] C. D. Diallo, O. Quevedo-Teruel, G. Valerio, H. Legay, and R. Sauleau, "Parallel-plate-waveguide Luneburg lens through a holey plate metasurface," in 2015 9th European Conference on Antennas and Propagation (EuCAP), May 2015, pp. 1-2.

[31] B. B. Tierney and A. Grbic, "A compact metamaterial beamformer designed through optimization," in Proceedings of the IEEE International Symposium on Antennas and Propagation, Jun. 2016, pp. 1-2.

[32] H. Bayer, A. Krauss, T. Zaiczek, R. Stephan, O. Enge-Rosenblatt, and M. A. Hein, "Ka-band user terminal antennas for satellite communications [antenna applications corner]," IEEE Antennas and Propagation Magazine, vol. 58, no. 1, pp. 76-88, Feb. 2016.

[33] O. Quevedo-Teruel, M. Ebrahimpouri, and F. Ghasemifard, "Lens antennas for 5G communications systems," IEEE Communications Magazine, special issue on Future 5G millimeter Wave Systems and Terminals, in press.

[34] M. Camacho, R. C. Mitchell-Thomas, A. P. Hibbins, J. R. Sambles, and O. Quevedo-Teruel, "Mimicking glide symmetry dispersion with coupled slot metasurfaces," Applied Physics Letters, vol. 111, no. 12, p. 121603, 2017. [Online]. Available: http://dx.doi.org/10.1063/1.5000222

[35] _ , "Designer surface plasmon dispersion on a one-dimensional periodic slot metasurface with glide symmetry," Opt. Lett., vol. 42, no. 17, pp. 3375-3378, Sep 2017. [Online]. Available: http://ol.osa.org/abstract.cfm?URI=ol-42-17-3375

[36] M. Ebrahimpouri, O. Quevedo-Teruel, and E. Rajo-Iglesias, "Design guidelines for gap waveguide technology based on glide-symmetric holey structures," IEEE Microwave and Wireless Components Letters, vol. 27, no. 6, pp. 542-544, June 2017.

[37] M. Ebrahimpouri, A. Algaba-Brazalez, L. Manholm, and O. QuevedoTeruel, "Using glide-symmetric holes to reduce leakage between waveguide flanges," IEEE Microwave and Wireless Components Letters, in press.

[38] Z. Sipus, H. Merkel, and P. S. Kildal, "Green's functions for planar soft and hard surfaces derived by asymptotic boundary conditions," IEE Proceedings - Microwaves, Antennas and Propagation, vol. 144, no. 5, pp. 321-328, Oct. 1997.

[39] M. Bosiljevac, Z. Sipus, and P. S. Kildal, "Construction of Green's functions of parallel plates with periodic texture with application to gap waveguides - a plane-wave spectral-domain approach," IET Microwaves, Antennas Propagation, vol. 4, no. 11, pp. 1799-1810, Nov. 2010.

[40] G. Valerio, Z. Sipus, A. Grbic, and O. Quevedo-Teruel, "Accurate equivalent-circuit descriptions of thin glide-symmetric corrugated metasurfaces," IEEE Transactions on Antennas and Propagation, vol. 65 no. 5, pp. 2695-2700, May 2017

[41] A. Wexler, "Solution of waveguide discontinuities by modal analysis," IEEE Transactions on Microwave Theory and Techniques, vol. 15, no. 9, pp. 508-517, September 1967.

[42] P. J. B. Clarricoats and K. R. Slinn, "Numerical method for the solution of waveguide-discontinuity problems," Electronics Letters, vol. 2, no. 6, pp. 226-228, June 1966.

[43] G. Valerio, Z. Sipus, A. Grbic, and O. Quevedo-Teruel, "Nonresonant modes in plasmonic holey metasurfaces for the design of artificial flat lenses," Opt. Lett., vol. 42, no. 10, pp. 2026-2029, May 2017. [Online]. Available: http://ol.osa.org/abstract.cfm?URI=ol-42-10-2026

[44] F. Ghasemifard, M. Ebrahimpouri, M. Norgren, and O. Quevedo-Teruel, "Mode matching analysis of two dimensional glide-symmetric corrugated metasurfaces," in 2017 11th European Conference on Antennas and Propagation (EUCAP), March 2017, pp. 749-751.

[45] F. Ghasemifard, M. Norgren, and O. Quevedo-Teruel, "Dispersion analysis of 2-d glide-symmetric corrugated metasurfaces using modematching technique," IEEE Microwave and Wireless Components Letters, vol. 28, no. 1, pp. 1-3, Jan 2018.

[46] "CST Microwave Studio," http://www.cst.com/, version: 2016.

[47] X. Dardenne and C. Craeye, "Method of moments simulation of infinitely periodic structures combining metal with connected dielectric objects," IEEE Transactions on Antennas and Propagation, vol. 56, no. 8, pp. 2372-2380, Aug 2008.

[48] G. Valerio, P. Baccarelli, S. Paulotto, F. Frezza, and A. Galli, "Regularization of mixed-potential layered-media green's functions for efficient interpolation procedures in planar periodic structures," IEEE Transactions on Antennas and Propagation, vol. 57, no. 1, pp. 122-134, Jan 2009.

[49] A. L. Fructos, R. R. Boix, and F. Mesa, "Application of kummer's transformation to the efficient computation of the 3-d green's function with 1-d periodicity," IEEE Transactions on Antennas and Propagation, vol. 58, no. 1, pp. 95-106, Jan 2010.

[50] G. Valerio, S. Paulotto, P. Baccarelli, D. R. Jackson, D. R. Wilton, W. A. Johnson, and A. Galli, "Efficient computation of 1-d periodic layered mixed potentials for the analysis of leaky-wave antennas with vertical elements," IEEE Transactions on Antennas and Propagation, vol. 63 , no. 6, pp. 2396-2411, June 2015.

[51] F. T. Celepcikay, D. R. Wilton, D. R. Jackson, and W. A. Johnson, "Interpolation of ewald-accelerated periodic green's function representations for homogeneous or layered media," IEEE Transactions on Antennas and Propagation, vol. 65, no. 5, pp. 2517-2525, May 2017.

[52] F. J. Garcia-Vidal, L. Martn-Moreno, and J. B. Pendry, "Surfaces with holes in them: new plasmonic metamaterials," Journal of Optics A: Pure and Applied Optics, vol. 7, no. 2, p. S97, 2005. [Online]. Available: http://stacks.iop.org/1464-4258/7/i=2/a=013

[53] V. Galdi and I. M. Pinto, "A simple algorithm for accurate location of leaky-wave poles for grounded inhomogeneous dielectric slabs," Microwave and Optical Technology Letters, vol. 24, no. 2, pp. 135-140, 2000.

[54] M. Ebrahimpouri and O. Quevedo-Teruel, "Bespoke lenses based on quasi-conformal transformation optics technique," IEEE Transactions on Antennas and Propagation, vol. 65, no. 5, pp. 2256-2264, May 2017.

[55] R. C. Mitchell-Thomas and O. Quevedo-Teruel, Transformation Optics Applied to Antennas and Focusing Systems. Cham: Springer International Publishing, 2018, pp. 387-406. 


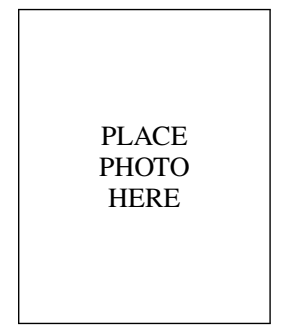

Guido Valerio (S'06-M'10) received the M.S. degree (cum laude and honorable mention) in electronic engineering in 2005, and the Ph.D. degree in electromagnetics in 2009, from La Sapienza University, Rome, Italy. From February to August 2008 he was a Visiting Scholar at the University of Houston, TX, USA. From 2011 to 2014, he was a researcher at the Institute d'Électronique et de Télécommunications de Rennes (IETR), France. Since September 2014 he is an Associate Professor in the Laboratoire d'Électronique and Électromagnétisme, at Sorbonne Université, Paris, France.

His scientific interests involve antenna design and numerical methods for wave propagation and scattering in complex structures; namely, periodic Greens function computation, modal properties of multilayered structures, full-wave methods for SIW, modeling of periodic structures with highersymmetries.

In 2008 Dr. Valerio was the recipient of the "Leopold B. Felsen Award for Excellence in Electrodynamics." In 2009 he was a finalist for the "Young Engineering Prize" at the European Microwave Conference. In 2010 he was the recipient of the Barzilai Prize for the best paper at the National Italian Congress of Electromagnetism (XVIII RiNEm). In 2014, he was the recipient of the RMTG Award for junior researchers presented at the IEEE Antennas and Propagation Society Symposium, Memphis, TN.

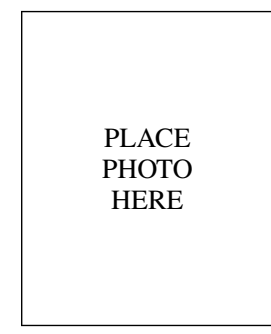

Fatemeh Ghasemifard received the B.Sc. degree in electrical engineering from Iran University of Science and Technology, Tehran, Iran and M.Sc. degree in electromagnetics engineering from University of Tehran, Tehran, Iran in 2011. In 2013, she joined KTH Royal Institute of Technology, Stockholm, Sweden, where she is currently a PhD student. Her main areas of research interest are metamaterials, metasurfaces, high-symmetric periodic structures, analytical and numerical methods in electromagnetics.

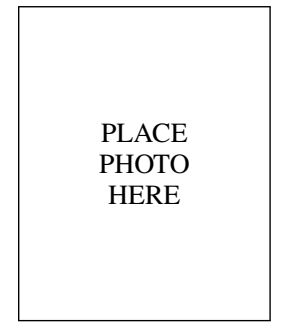

Zvonimir Sipus (M'98-SM'13) was born in Zagreb, Croatia, in 1964. He received the B.Sc. and M.Sc. degrees in electrical engineering from the University of Zagreb, Croatia, in 1988 and 1991, respectively, and the Ph.D. degree in electrical engineering from the Chalmers University of Technology, Gothenburg, Sweden, in 1997. From 1988 to 1994, he was with the Rudjer Boskovic Institute, Zagreb, Croatia, as a Research Assistant, where he was involved in the development of detectors for explosive gases. In 1994, he joined the Antenna Group, Chalmers University of Technology, where he was involved in research projects concerning conformal antennas and soft and hard surfaces. In 1997, he joined the Faculty of Electrical Engineering and Computing, University of Zagreb, where he is currently a Professor. From 2008 to 2012 and since 2014, he has been the Head of the Department of Wireless Communications. From 1999 to 2005, he was also an Adjunct Researcher with the Department of Electromagnetics, Chalmers University of Technology. Since 2006, he has been involved in teaching with the European School of Antennas.

His current research interests include analysis and design of electromagnetic structures with application to antennas, microwaves, and optical communication and sensor systems.

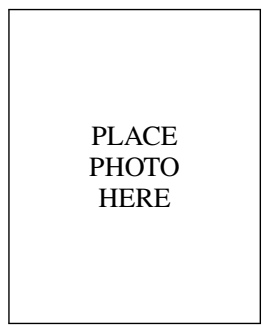

Oscar Quevedo Teruel (M'10-SM'17) received his M.Sc. degree in Telecommunication Engineering from Carlos III University of Madrid Spain in 2005, and went on to complete his Master of Engineering at Chalmers University of Technology in Sweden. He obtained his PhD from Carlos III University of Madrid in 2010 and was then invited into a postdoctoral research position at the University of Delft (The Netherlands). From 2010-2011, Dr. QuevedoTeruel joined the Department of Theoretical Physics of Condensed Matter at Universidad Autonoma de Madrid as a research fellow and went on to continue his postdoctoral research at Queen Mary University of London from 2011-2013. In 2014, he joined the School of Electrical Engineering (EES)/Electromagnetic Engineering (ETK) at KTH Royal Institute of Technology in Stockholm Sweden where he is an Associate Professor.

He has made scientific contributions in the fields of microstrip patch antennas, leaky-wave antennas, lens antennas, metasurfaces with higher symmetries, transformation optics and high impedance surfaces.

$\mathrm{He}$ is the co-author of more than 54 papers in international journals, more than 110 at international conferences, and has received approval on 2 patents. 